\title{
Urtica dioica-Derived Phytochemicals for Pharmacological and Therapeutic Applications
}

\author{
Yasaman Taheri $\mathbb{D}^{1},{ }^{1}$ Cristina Quispe, ${ }^{2}$ Jesús Herrera-Bravo ${ }^{D},{ }^{3,4}$ Javad Sharifi-Rad $\mathbb{D},{ }^{1,5}$ \\ Shahira M. Ezzat $\mathbb{D}^{\mathrm{D}},{ }^{6,7}$ Rana M. Merghany, ${ }^{8}$ Shabnum Shaheen, ${ }^{9}$ Lubna Azmi, ${ }^{10}$ \\ Abhay Prakash Mishra ${ }^{\mathbb{D}},{ }^{11}$ Bilge Sener, ${ }^{12}$ Mehtap Kılıç, ${ }^{13}$ Surjit Sen, ${ }^{14,15}$ \\ Krishnendu Acharya, ${ }^{14}$ Azadeh Nasiri, ${ }^{16}$ Natália Cruz-Martins $\mathbb{D},{ }^{17,18,19,20}$ \\ Patrick Valere Tsouh Fokou ${ }^{(D,}{ }^{21}$ Alibek Ydyrys, ${ }^{22}$ Zhandos Bassygarayev, ${ }^{23}$ \\ Sevgi Durna Daştan, ${ }^{24,25}$ Mohammed M. Alshehri $\mathbb{D}^{26}{ }^{26}$ Daniela Calina $\mathbb{D}^{27}$ \\ and William C. Cho ${ }^{28}$
}

\footnotetext{
${ }^{1}$ Phytochemistry Research Center, Shahid Beheshti University of Medical Sciences, Tehran, Iran

${ }^{2}$ Facultad de Ciencias de la Salud, Universidad Arturo Prat, Avda. Arturo Prat 2120, Iquique 1110939, Chile

${ }^{3}$ Departamento de Ciencias Básicas, Facultad de Ciencias, Universidad Santo Tomas, Chile

${ }^{4}$ Center of Molecular Biology and Pharmacogenetics, Scientific and Technological Bioresource Nucleus,

Universidad de La Frontera, Temuco, 4811230, Chile

${ }^{5}$ Facultad de Medicina, Universidad del Azuay, Cuenca, Ecuador

${ }^{6}$ Department of Pharmacognosy, Faculty of Pharmacy, Cairo University, Kasr El Ainy Street, Cairo 11562, Egypt

${ }^{7}$ Department of Pharmacognosy, Faculty of Pharmacy, October University for Modern Sciences and Arts (MSA), 6th of October 12451, Egypt

${ }^{8}$ Department of Pharmacognosy, National Research Centre, Giza, Egypt

${ }^{9}$ Department of Plant Sciences, LCWU, Lahore 54000, Pakistan

${ }^{10}$ Hygia Institute of Pharmaceutical Education \& Research, Lucknow, U. P. 226001, India

${ }^{11}$ Department of Pharmacology, University of Free State, Bloemfontein 9300, Free State, South Africa

${ }^{12}$ Gazi University, Faculty of Pharmacy, Department of Pharmacognosy, Ankara 06330, Turkey

${ }^{13}$ Department of Pharmacognosy, Lokman Hekim University Faculty of Pharmacy, Ankara 06510, Turkey

${ }^{14}$ Molecular and Applied Mycology and Plant Pathology Laboratory, Department of Botany, University of Calcutta, Kolkata 700019, India

${ }^{15}$ Department of Botany, Fakir Chand College, Diamond Harbour, West Bengal 743331, India

${ }^{16}$ Department of Pharmacology and Toxicology, School of Pharmacy, Shahid Beheshti University of Medical Sciences, Tehran, Iran

${ }^{17}$ Faculty of Medicine, University of Porto, Porto, Portugal

${ }^{18}$ Institute for Research and Innovation in Health (i3S), University of Porto, Porto, Portugal

${ }^{19}$ Institute of Research and Advanced Training in Health Sciences and Technologies (CESPU), Rua Central de Gandra, 1317, Gandra PRD 4585-116, Portugal

${ }^{20}$ TOXRUN-oxicology Research Unit, University Institute of Health Sciences, CESPU, CRL, Gandra 4585-116, Portugal

${ }^{21}$ Department of Biochemistry, Faculty of Science, University of Bamenda, Bambili, P.O. Box. 39, Cameroon

${ }^{22}$ Biomedical Research Centre, Al-Farabi Kazakh National University, Al-Farabi av. 71, Almaty 050040, Kazakhstan

${ }^{23}$ Department of Biophysics, Biomedicine and Neuroscience, Al-Farabi Kazakh National University, Al-Farabi av. 71, Almaty 050040, Kazakhstan

${ }^{24}$ Department of Biology, Faculty of Science, Sivas Cumhuriyet University, Sivas 58140, Turkey

${ }^{25}$ Beekeeping Development Application and Research Center, Sivas Cumhuriyet University, Sivas 58140, Turkey

${ }^{26}$ Pharmaceutical Care Department, Ministry of National Guard-Health Affairs, Riyadh, Saudi Arabia

${ }^{27}$ Department of Clinical Pharmacy, University of Medicine and Pharmacy of Craiova, Craiova 200349, Romania

${ }^{28}$ Department of Clinical Oncology, Queen Elizabeth Hospital, Kowloon, Hong Kong
}

Correspondence should be addressed to Javad Sharifi-Rad; javad.sharifirad@gmail.com, Shahira M. Ezzat; shahira.ezzat@ pharma.cu.edu.eg, and Patrick Valere Tsouh Fokou; ptsouh@gmail.com 
Received 12 October 2021; Accepted 7 January 2022; Published 24 February 2022

Academic Editor: Lu sa Mota da Silva

Copyright (C) 2022 Yasaman Taheri et al. This is an open access article distributed under the Creative Commons Attribution License, which permits unrestricted use, distribution, and reproduction in any medium, provided the original work is properly cited.

Urtica dioica belongs to the Urticaceae family and is found in many countries around the world. This plant contains a broad range of phytochemicals, such as phenolic compounds, sterols, fatty acids, alkaloids, terpenoids, flavonoids, and lignans, that have been widely reported for their excellent pharmacological activities, including antiviral, antimicrobial, antihelmintic, anticancer, nephroprotective, hepatoprotective, cardioprotective, antiarthritis, antidiabetic, antiendometriosis, antioxidant, anti-inflammatory, and antiaging effects. In this regard, this review highlights fresh insight into the medicinal use, chemical composition, pharmacological properties, and safety profile of $U$. dioica to guide future works to thoroughly estimate their clinical value.

\section{Introduction}

Genus Urtica, commonly known as "nettle," is a medicinal plant belonging to the family Urticaceae with multiple health benefits that have been used medicinally since at least the times of Ancient Greece [1]. Several Urtica species have been widely used to treat rheumatism and sciatica, asthma, coughs, dandruff, diabetes, diarrhea, eczema, fever, gout, hemorrhoids, nose bleeds, scurvy, snake bites, and tuberculosis [2]. Moreover, Urtica species have been used most commonly as a diuretic and for treating gout, anemia, and prostate hypertrophy, with several studies progressively reporting their traditional medicinal use by local people [2-11].

These studies originated mostly from African, European, Asian, and Oceanian countries, such as Algeria, Argentina, Australia, Bolivia, Bhutan, Brazil, Bolivia, Belarus, Bolivia, Canada, Chile, China, Colombia, Cyprus, Costa Rica, Cuba, Ecuador, Egypt, France, Guatemala, India, Italy, Israel, Japan, Korea, Mexico, Nepal, New Zealand, Netherlands, North America, Palestine, Paraguay, Peru, Russia, Sikkim, Sweden, Spain, Taiwan, Turkey, Tunisia, United States, Uruguay, Ukraine, and Vietnam [2-11].

Data obtained from these studies mostly underlined that Urtica species exert excellent antirheumatoid arthritis, antigout, anti-inflammatory, immunomodulatory, and antioxidant activities, all of which contribute to the protection of joints. In addition, it has been revealed to be extremely useful for the treatment of microbial and parasitic infections, cancer, jaundice, stomach diseases, snakebites, diabetes, liver and kidney problems, wounds, diuretic, libido, pulmonary diseases, hypotensive, blood purification, urticaria, allergic rhinitis, prostate disorders, hemorrhoids, and galactagogue and as a depurative. Apart from this, these species have also been reported to be used for exorcism, postcalving care, sprains, bones fracture, hematuria, neck sore, and yolk sore [2, 12-26].

Despite the scientific advances that have allowed us to understand the crucial contribution of the active molecules present in this plant for their biological and therapeutic potentialities, the relevance of this knowledge goes beyond chemical features, as it is necessary to understand that due to the increased daily living standards of rural populations, decisions regarding the sustainable use of plant resources have been even more underlined $[27,28]$. In this sense, this review aims to provide an overview of the botanical features, chemical composition, and biological effects of Urtica species towards well-being promotion and disease prevention.

\section{Botanical Features and Geographical Location}

Urtica species is a nitrophilous plant that can grow up to 1$2 \mathrm{~m}$ in height depending on edaphic conditions. Despite growing well in areas with high water availability [29-31], the plant can spread widely with its stoloniferous rhizomes [32].

Leaves are simple, dark green, stipulate, opposite, serrated, oblong, or ovate with cordate base $[33,34]$. Both leaves surfaces are coated with stinging hairs; except in the European variety (Urtica galeopsifolia), the stinging hairs are absent [35]. Stem is green, erect, hollow to solid, fibrous and tough, indumentum of many stinging hairs and trichomes.

Flowers are small, reddish-brown to greenish-white in colour, mostly dioecious occurring as racemes in the axial of the upper leaves; staminate flowers with 4-5 long tepals, stamens 4, exserted, filaments flat; pistillate flowers with 4 short tepals, sparsely pubescent, esetulose, ovary superior, ovoid, 1-celled [1, 32, 34].

Urtica species present a subcosmopolitan distribution, being found around the globe, except in Antarctica and some tropical regions $[36,37]$. The plant is commonly found as a weed, mainly in moist and shady places and often in anthropogenic habitats. The genus comprises 46 species, being the most important Urtica dioica (stinging nettle) and Urtica urens (small nettle), which are native to Europe, Africa, Asia, North America, and naturalized in other temperate parts of the world (Table 1) [2, 5].

The widely distributed weedy species, $U$. dioica, is considered an ecological keystone species and, thus, it is significantly important for the biodiversity in the ecosystem [38-42]. Island endemics are very common within this genus and the species include $U$. dioica subsp. cypria on Cyprus island, Urtica atrovirens on Corsica and Sardinia, Urtica rupestris on Sicily, Urtica stachyoides on the Canary Islands, Urtica portosanctana on Madeira, Urtica bianorii on Mallorca, Urtica domingensis on Hispaniola, Urtica glomerulaeflora on Juan Fernández Islands, Urtica grandidentata on Indonesia, Urtica taiwaniana on Taiwan, Urtica papuana on 
TABLE 1: Geographical distribution, traditional uses, and pharmacology of Urtica species.

\begin{tabular}{|c|c|c|c|c|}
\hline No. & Species & $\begin{array}{l}\text { Geographical } \\
\text { distribution }\end{array}$ & Traditional uses & Pharmacological activities \\
\hline 1 & Urtica andicola Wedd. & Turkey & Skin rashes, arthritis, fungal infections & - \\
\hline 2 & $\begin{array}{l}\text { Urtica angustifolia } \\
\text { Fisch. ex Hornem. }\end{array}$ & $\begin{array}{l}\text { China, Japan, Korea, } \\
\text { Mongolia, Siberia }\end{array}$ & None known & Antifatigue \\
\hline 3 & Urtica ardens Link & $\begin{array}{l}\text { Bhutan, India, Nepal, } \\
\text { Sikkim }\end{array}$ & $\begin{array}{c}\text { Exorcism, jaundice, postcalving care, } \\
\text { sprains, bones fracture, hematuria, neck } \\
\text { sore, yolk sore }\end{array}$ & - \\
\hline 4 & Urtica aspera Petrie & New Zealand & $\begin{array}{l}\text { Stomach diseases, snakebites, } \\
\text { inflammation, rheumatoid arthritis, } \\
\text { hyperplasia, fungal infections }\end{array}$ & - \\
\hline 5 & $\begin{array}{l}\text { Urtica atrichocaulis } \\
\text { (Hand.-Mazz.) C.J. } \\
\text { Chen }\end{array}$ & $\begin{array}{l}\text { China, Japan, Korea, } \\
\text { Himalayas, Pakistan }\end{array}$ & $\begin{array}{l}\text { Rheumatoid arthritis, inflammatory, } \\
\text { antioxidant, immune-modulatory }\end{array}$ & - \\
\hline 6 & $\begin{array}{l}\text { Urtica atrovirens Req. } \\
\text { ex Loisel }\end{array}$ & France, Italy, Spain & $\begin{array}{c}\text { Antihyperglycemic, antioxidant, hepatic } \\
\text { protective, antiviral, arthritis }\end{array}$ & - \\
\hline 7 & $\begin{array}{l}\text { Urtica australis } \\
\text { Hook.f. }\end{array}$ & New Zealand & $\begin{array}{l}\text { Skin diseases, diabetes, eczema, fungal } \\
\text { infections, arthritis }\end{array}$ & - \\
\hline 8 & $\begin{array}{l}\text { Urtica ballotifolia } \\
\text { Wedd. }\end{array}$ & Colombia, Ecuador & - & - \\
\hline 9 & Urtica berteroana Phil & $\begin{array}{l}\text { Chile, Bolivia, } \\
\text { Argentina, Colombia }\end{array}$ & - & - \\
\hline 10 & Urtica buchtienii Ross & $\begin{array}{l}\text { Chile, Argentina } \\
\text { Russia, Sweden, }\end{array}$ & - & - \\
\hline 11 & Urtica cannabina $\mathrm{L}$. & $\begin{array}{l}\text { Netherlands, China, } \\
\text { Western Asia from } \\
\text { Siberia to Iran }\end{array}$ & - & Anti-inflammatory \\
\hline 12 & $\begin{array}{c}\text { Urtica chamaedryoides } \\
\text { Pursh }\end{array}$ & United States, Mexico & - & - \\
\hline 13 & Urtica circularis Sorarú & $\begin{array}{l}\text { Brazil, Argentina, } \\
\text { Paraguay, Uruguay }\end{array}$ & - & Antioxidant, anti-inflammatory \\
\hline 14 & Urtica deltoidea Sw. & New Zealand & $\begin{array}{l}\text { Arthritis, inflammation, antiulcer, } \\
\text { anticancer, antimicrobial activities }\end{array}$ & - \\
\hline 15 & $\begin{array}{l}\text { Urtica dentate Hand.- } \\
\text { Mazz }\end{array}$ & North America & $\begin{array}{l}\text { Kidney problems, rheumatoid arthritis, } \\
\text { kidney calculi }\end{array}$ & Antiarthritis, antiurolithiatic \\
\hline 16 & Urtica dioica $\mathrm{L}$. & $\begin{array}{l}\text { United States, New } \\
\text { Zealand, Turkey, } \\
\text { Europe, Asia, North } \\
\text { America }\end{array}$ & $\begin{array}{l}\text { Injuries to reduce swelling, diuretic, flu, } \\
\text { diabetes disease, losing weight, cold, } \\
\text { cancers, anemic conditions, libido, } \\
\text { induce menstruation, stomach- ache, } \\
\text { renal and pulmonary diseases }\end{array}$ & $\begin{array}{l}\text { Antiviral, antimicrobial, antioxidant, } \\
\text { anti-inflammatory antiaging, cytotoxic/ } \\
\text { anticancer Effect on benign prostatic } \\
\text { hyperplasia, antidiabetic, } \\
\text { antiendometriosis, nephroprotective }\end{array}$ \\
\hline 17 & Urtica echinata Benth & $\begin{array}{c}\text { Bolivia, Peru, } \\
\text { Argentina, Ecuador }\end{array}$ & - & - \\
\hline 18 & Urtica ferox Blanco & New Zealand, Australia & $\begin{array}{l}\text { Skin problems, hyperglycemic, antiviral, } \\
\text { diuretic, hypotensive, antiaggregate }\end{array}$ & - \\
\hline 19 & Urtica fissa E. Pritz & $\begin{array}{c}\text { China, Taiwan, Egypt, } \\
\text { Vietnam }\end{array}$ & Rheumatoid arthritis & - \\
\hline 20 & Urtica flabellata Kunth & $\begin{array}{l}\text { Bolivia, Peru, Ecuador, } \\
\text { Chile, Colombia, } \\
\text { Turkey }\end{array}$ & Skin rashes, arthritis, fungal infections & - \\
\hline 21 & $\begin{array}{l}\text { Urtica galeopsifolia } \\
\text { J. Jacq. ex Blume }\end{array}$ & Russia, Ukraine, Belarus & $\begin{array}{l}\text { Renal ailments, asthma, anemia, blood } \\
\text { purification }\end{array}$ & - \\
\hline 22 & $\begin{array}{l}\text { Urtica gracilenta } \\
\text { Greene }\end{array}$ & & $\begin{array}{c}\text { Kidney diseases, diabetes, fungal } \\
\text { infections }\end{array}$ & - \\
\hline 23 & $\begin{array}{l}\text { Urtica glomeruliflora } \\
\text { Steud. }\end{array}$ & Chile & & - \\
\hline 24 & $\begin{array}{l}\text { Urtica haussknechtii } \\
\text { Boiss. }\end{array}$ & Turkey & & - \\
\hline 25 & $\begin{array}{l}\text { Urtica hyperborea Jacq. } \\
\text { exWedd. }\end{array}$ & Nepal, India, China & Skin rashes, arthritis, fungal infections & Antioxidant \\
\hline 26 & Urtica incana Blume & Peru & Skin rashes, arthritis, fungal infections & - \\
\hline
\end{tabular}


TABle 1: Continued.

\begin{tabular}{|c|c|c|c|c|}
\hline No. & Species & $\begin{array}{l}\text { Geographical } \\
\text { distribution }\end{array}$ & Traditional uses & Pharmacological activities \\
\hline 27 & $\begin{array}{l}\text { Urtica kioviensis } \\
\text { Rogow. }\end{array}$ & Europe, Israel, Russia & Arthritis, hepatic protective, antiviral & - \\
\hline 28 & $\begin{array}{l}\text { Urtica lalibertadensis } \\
\text { Weigend }\end{array}$ & Peru & Skin rashes, arthritis, fungal infections & - \\
\hline 29 & $\begin{array}{l}\text { Urtica laetevirens } \\
\text { Maxim. }\end{array}$ & China, Japan, Korea & & Anticancer \\
\hline 30 & $\begin{array}{l}\text { Urtica leptophylla } \\
\text { Kunth }\end{array}$ & $\begin{array}{l}\text { Costa Rica, Colombia, } \\
\text { Peru, Bolivia, Ecuador }\end{array}$ & Skin rashes, arthritis, fungal infections & - \\
\hline 31 & $\begin{array}{l}\text { Urtica lilloi (Hauman) } \\
\text { Geltman }\end{array}$ & Argentina & & - \\
\hline 32 & Urtica longispica Killip & $\begin{array}{l}\text { Ecuador, Peru, } \\
\text { Colombia }\end{array}$ & $\begin{array}{l}\text { Cough, eczema, gout, urticaria, allergic } \\
\text { rhinitis, rheumatoid arthritis }\end{array}$ & - \\
\hline 33 & Urtica macbridei Killip & Ecuador, Peru & & - \\
\hline 34 & $\begin{array}{l}\text { Urtica magellanica } \\
\text { Juss. ex Poir. }\end{array}$ & $\begin{array}{l}\text { Chile, Peru, Bolivia, } \\
\text { Argentina, Ecuador }\end{array}$ & Allergy, arthritis & - \\
\hline 35 & Urtica mairei H. Lév. & $\begin{array}{l}\text { China, India, Bhutan, } \\
\text { Himalaya, Myanmar }\end{array}$ & $\begin{array}{l}\text { Kidney pain. Its extract and paste kidney } \\
\text { diseases, diabetes, fungal infections, } \\
\text { inflammation, arthritis }\end{array}$ & Antiprotatic hyperplasia \\
\hline 36 & $\begin{array}{c}\text { Urtica masafuerae } \\
\text { Phil. }\end{array}$ & Chile & & - \\
\hline 37 & $\begin{array}{l}\text { Urtica massaica } \\
\text { Mildbr. }\end{array}$ & Africa & $\begin{array}{l}\text { Skin rashes, malaria, eczema, skin } \\
\text { rashes, dermatitis, diuretic }\end{array}$ & - \\
\hline 38 & $\begin{array}{l}\text { Urtica membranacea } \\
\text { Poir. ex Savigny }\end{array}$ & Israeli, Europe, Algeria & - & Antioxidant, anti-inflammatory \\
\hline 39 & Urtica mexicana Liebm & Mexico, Guatemala & - & - \\
\hline 40 & Urtica mollis Steud. & Peru, Chile, Argentina & - & - \\
\hline 41 & Urtica morifolia Poir. & Europe & - & - \\
\hline 42 & Urtica orizabae Liebm. & $\begin{array}{l}\text { Mexico, United States, } \\
\text { Cuba }\end{array}$ & - & - \\
\hline 43 & Urtica parviflora Roxb. & $\begin{array}{l}\text { Nepal, India, United } \\
\text { States, Western China, } \\
\text { Bhutan, Himalaya }\end{array}$ & $\begin{array}{l}\text { Arthritis, tumor, astringent, diuretic, } \\
\text { inflammatory }\end{array}$ & $\begin{array}{c}\text { Nephroprotective, antidiabetic, } \\
\text { antioxidant }\end{array}$ \\
\hline 44 & Urtica pilulifera L. & $\begin{array}{l}\text { Tunisia, Israel, Cyprus, } \\
\text { Costa Rica, Turkey, } \\
\text { Palestine }\end{array}$ & $\begin{array}{l}\text { Skin and prostate disorders, rheumatoid } \\
\text { arthritis, diabetes, skin treatment, } \\
\text { inflammation, arthritis, internal } \\
\text { bleeding, anemia, excessive } \\
\text { menstruation, hemorrhoids, } \\
\text { rheumatism, hay fever, kidney } \\
\text { problems, pain, skin problems, } \\
\text { abdominal pain, internal diseases, } \\
\text { antiasthmatic, antitumor, astringent, } \\
\text { diuretic, galactagogue, depurative, } \\
\text { antihyperglycemic, antidandruff }\end{array}$ & Antidiabetic \\
\hline 45 & $\begin{array}{l}\text { Urtica platyphylla } \\
\text { Wedd. }\end{array}$ & Japan, Russia & - & - \\
\hline 46 & $\begin{array}{l}\text { Urtica praetermissa } \\
\text { V.W. Steinm. }\end{array}$ & Mexico & - & - \\
\hline 47 & $\begin{array}{l}\text { Urtica pubescens } \\
\text { Ledeb. }\end{array}$ & Mexico & - & - \\
\hline 48 & $\begin{array}{l}\text { Urtica rupestris Guss. } \\
\text { Urtica sondenii }\end{array}$ & Italy & - & - \\
\hline 49 & $\begin{array}{c}\text { (Simmons) Avrorin ex } \\
\text { Geltman }\end{array}$ & Canada & - & - \\
\hline 50 & $\begin{array}{c}\text { Urtica spiralis Blume } \\
\text { Urtica }\end{array}$ & Mexico & - & - \\
\hline 51 & $\begin{array}{l}\text { stachyoidesWebb \& } \\
\text { Benth. }\end{array}$ & Spain, Mexico & - & - \\
\hline 52 & $\begin{array}{l}\text { Urtica taiwaniana S.S. } \\
\text { Ying }\end{array}$ & Taiwan & - & - \\
\hline
\end{tabular}


TABLE 1: Continued.

\begin{tabular}{|c|c|c|c|c|}
\hline No. & Species & $\begin{array}{c}\text { Geographical } \\
\text { distribution }\end{array}$ & Traditional uses & Pharmacological activities \\
\hline 53 & $\begin{array}{l}\text { Urtica thunbergiana } \\
\text { Siebold \& Zucc. }\end{array}$ & Japan, Korea, China & - & Antiaging \\
\hline 54 & $\begin{array}{c}\text { Urtica triangularis } \\
\text { Hand.-Mazz. }\end{array}$ & China & - & - \\
\hline 55 & $\begin{array}{c}\text { Urtica trichantha } \\
\text { (Wedd.) Acevedo \& } \\
\text { Navas }\end{array}$ & $\begin{array}{l}\text { Chile, Bolivia, Peru, } \\
\text { Japan, China }\end{array}$ & - & - \\
\hline 56 & Urtica urens L. & $\begin{array}{l}\text { Unite States, Mexico, } \\
\text { Europe, Israel, New } \\
\text { Zealand }\end{array}$ & $\begin{array}{l}\text { Blood depurative, antihypoglycemic, } \\
\text { antioxidant, hepatic protective, antiviral, } \\
\text { diuretic, hypotensive, antiaggregate, } \\
\text { kidney problems }\end{array}$ & Antioxidant, anti-inflammatory \\
\hline
\end{tabular}

Papua New Guinea, and Urtica perconfusa on New Zealand. This indicates that island colonization within the genus is a unique feature amongst the flowering plants $[3,4]$.

\section{Phytoconstituents}

Phytochemicals are plant metabolites produced in response to any infectious attack or as a byproduct of any metabolic pathway, despite exerting beneficial effects in many ways $[43,44]$. The active chemical part of nettle includes nearly fifty compounds of the lipophilic and hydrophilic fractions and whose chemical structure is known. Globally, few Urtica species have been screened for their phytochemical composition, with those available so far reporting the presence of sterols, triterpenes, coumarins, phenols, lignans, ceramides, and fatty acids, amongst other minor compounds, all with a distribution varying in the various organs of the plant (Tables 2-11).

Beta-sitosterol, transferulic acid, dotriacontane, erucic acid, ursolic acid, scopoletin, rutin, quercetin, and phydroxylbenzalcohol are some of the constituents found in Urtica species that may be applied for preventive or therapeutic purposes in communicable and noncommunicable diseases [16, 45-59]. The liquid contained in the hairs of a nettle causes it to sting, being composed of formic acid and leukotrienes in modest amounts, 1\% acetylcholine, 1 in 500 to 1 in 2000 histamine, and 5-hydroxy-tryptamine (serotonin).

Essential ketones (38.5\%), esters (14.7\%), free alcohols (2\%), nitrogenous compounds, phenols, aldehydes, p-sitosterol, formic acid and acetic acid, chlorophyll and phytol, vitamins, and carotenoids are also found in the aerial sections. Many organic acids were also identified in the aerial parts, including caffeic, ferulic, caffeylmalic, chlorogenic, and sinapic acids, according to chromatographic examination.

Flavonoids: isorhamnetol 3-O-glucoside, quercetol 3-Oglucoside, kaempferol 3-O-glucoside, isorhamnetol 3-Orutinoside, and quercetol 3-O-rutinoside were extracted and identified in flowers, in addition to p-sitosterol, p-sitosterol glucoside, and scopoletol, which are found in all sections of the plant. The roots contained many molecules belonging to different chemical families, including polysaccharides: glycans, glucogalacturonans, arabinogalactan acid, fatty acid:
(10E, 12Z)-9-hydroxy-10, 12-octadecadienoic acid, lectins, ceramides, terpenes diols, and terpenes diols glucosides [60].

Amongst Urtica species, Urtica pilulifera and U. dioica essential oil compositions have been investigated and consist mainly of hexahydrofarnesyl acetone, 1,8-cineole, $\alpha$-ionone, $\beta$-ionone, farnesylacetone, methylbenzene, (-)-limonene, 3carene, (+)-limonene, gamma-terpinene, vanillin, butyl acetate, 1, 2-benzenedicarboxylic acid, and 7-acetyl-6-ethyl1, 1, 4, 4-tetramethyltetralin (Table 12) [61, 62]. Overall, considerably less attention has been paid to the phytochemistry of bioactive compounds in these plants.

\section{Pharmacological Activities of the Genus Urtica}

Except for $U$. dioica, which has extensively been studied for various pharmacological properties, few Urtica species have been investigated for their biological activity, including $U$. angustifolia, $U$. laetivirens, $U$. parviflora, $U$. dentata, $U$. pilulifera, $U$. mairei, $U$. membranacea, $U$. urens, $U$. circularis, $U$. hyperborean, $U$. cannabina, and $U$. thunbergiana that mostly displayed anti-inflammatory and antioxidant activities (Tables 1-13), and for antiviral, antimicrobial, antihelmintic, anticancer, nephroprotective, hepatoprotective, cardioprotective, antiarthritis, antidiabetic, antiendometriosis, and antiaging purposes (Figure 1)

\subsection{In Vitro Pharmacological Findings}

4.1.1. Antiviral Activity. Antiviral treatment is limited to severe cases of most viral infections, stressing the need for more effective therapy. The aqueous extract of $U$. dioica fresh bark showed an antiviral effect against Petaluma virus (FIVPet) that infected Crandell feline kidney cell line (CrFK) by significantly inhibiting viral replication through reducing syncytia formation at low doses $(0.5-1 \mathrm{~g} / \mathrm{ml})$ in a dose-dependent manner [73].

$U$. dioica extract $(0.5-1 \mathrm{~g} / \mathrm{ml})$ and derived $\mathrm{N}$-acetyl glucosamine-specific lectin (the $50 \%$ effective concentration $\left(\mathrm{EC}_{50}\right)$ for HIV ranged from 0.3 to $9 \mathrm{mg} / \mathrm{ml}$ ) also revealed to be able to inhibit syncytium synthesis between CD4+ MOLT/4 cells and HUT-78 cells when infected by HIV-1 and HIV-2 (Uncini Manganelli, Zaccaro \& Tomei, 2005). 


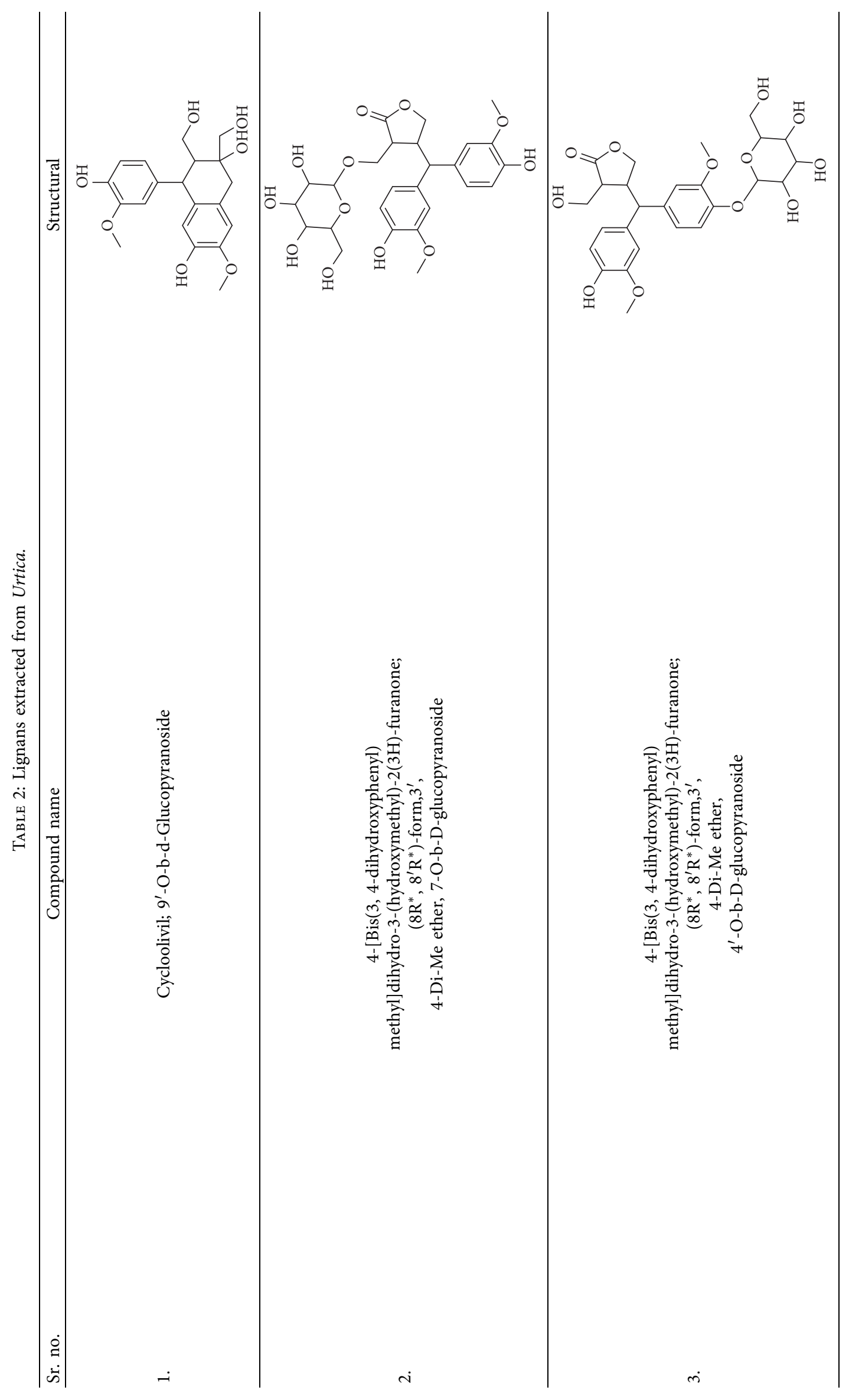




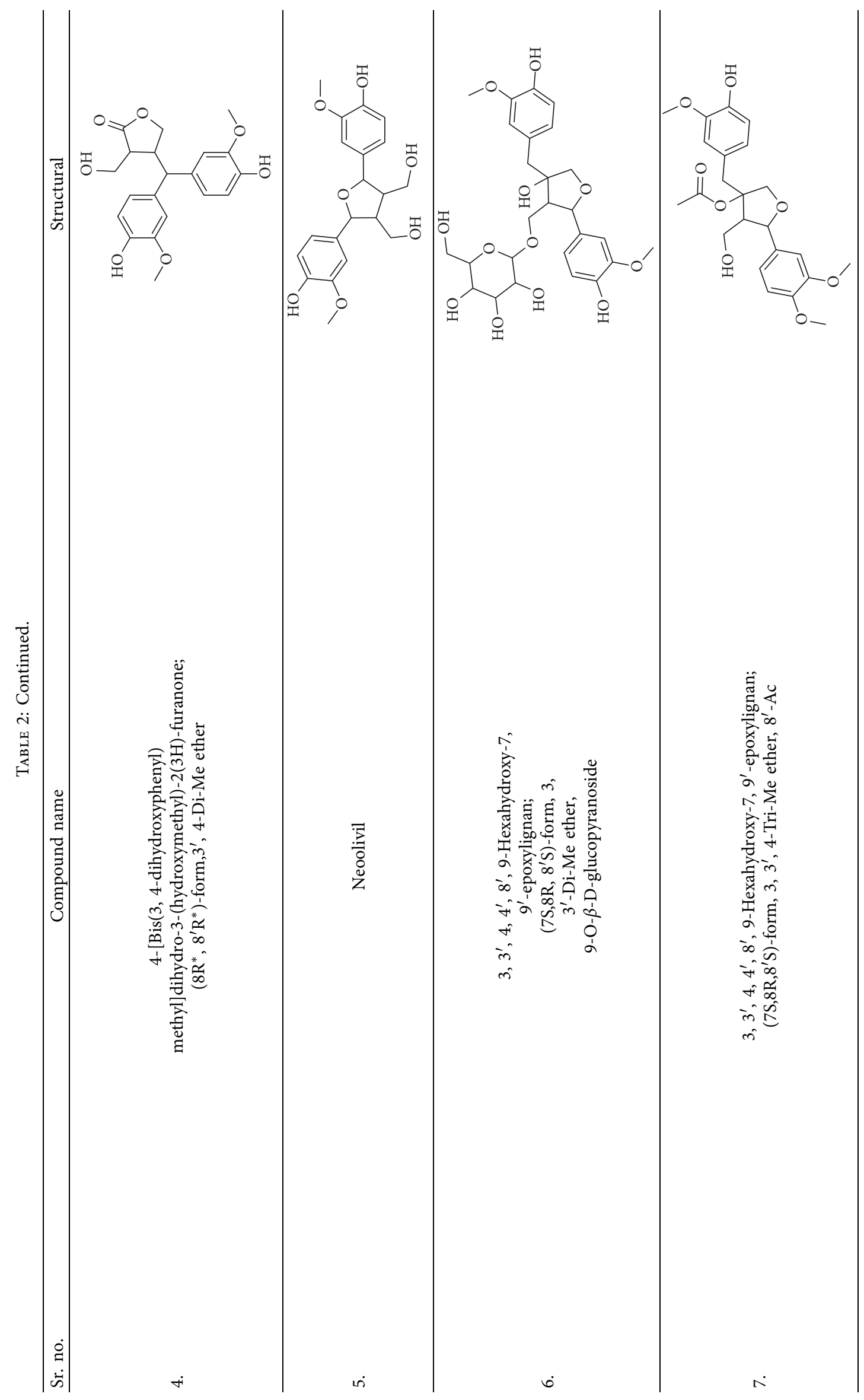




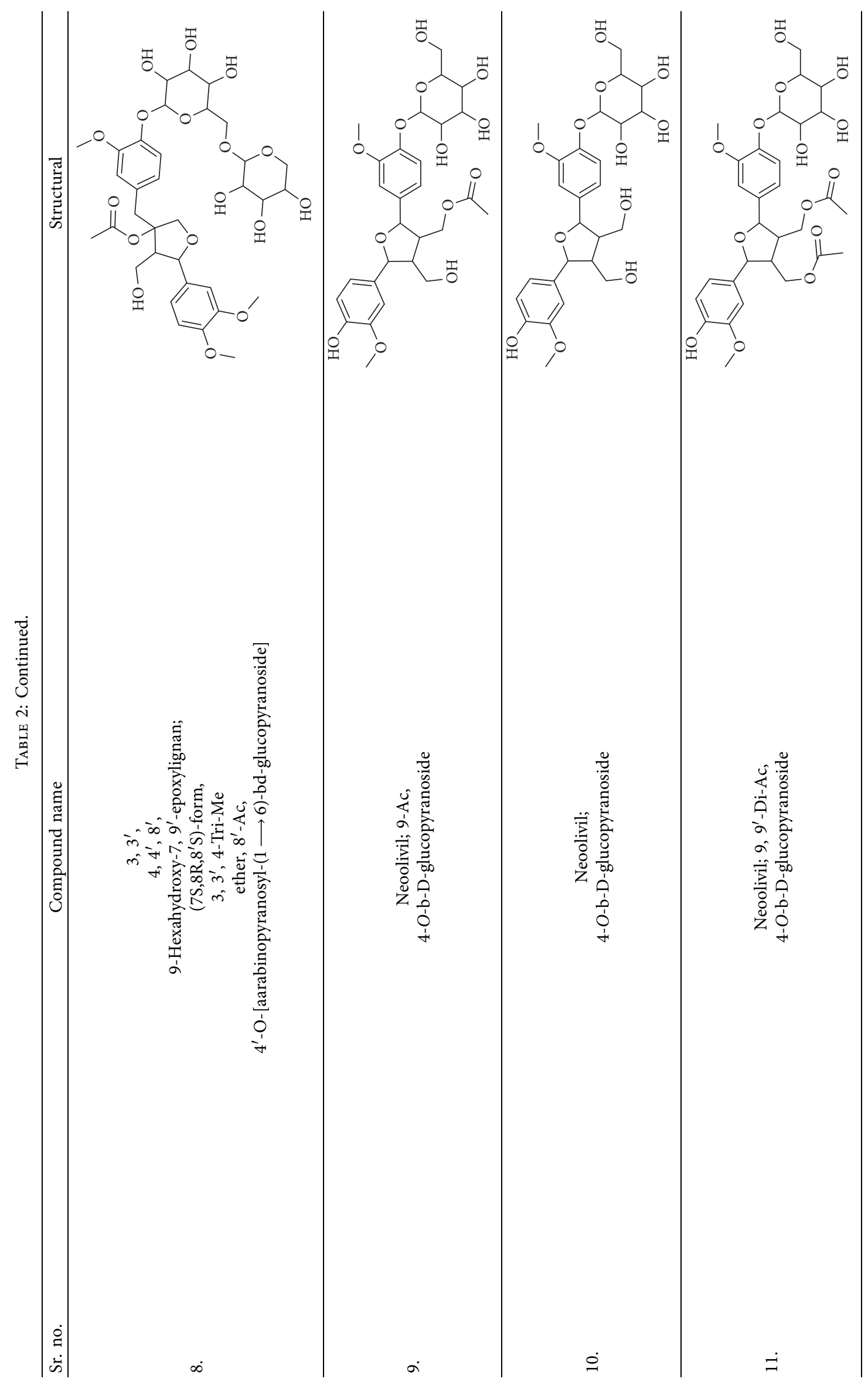




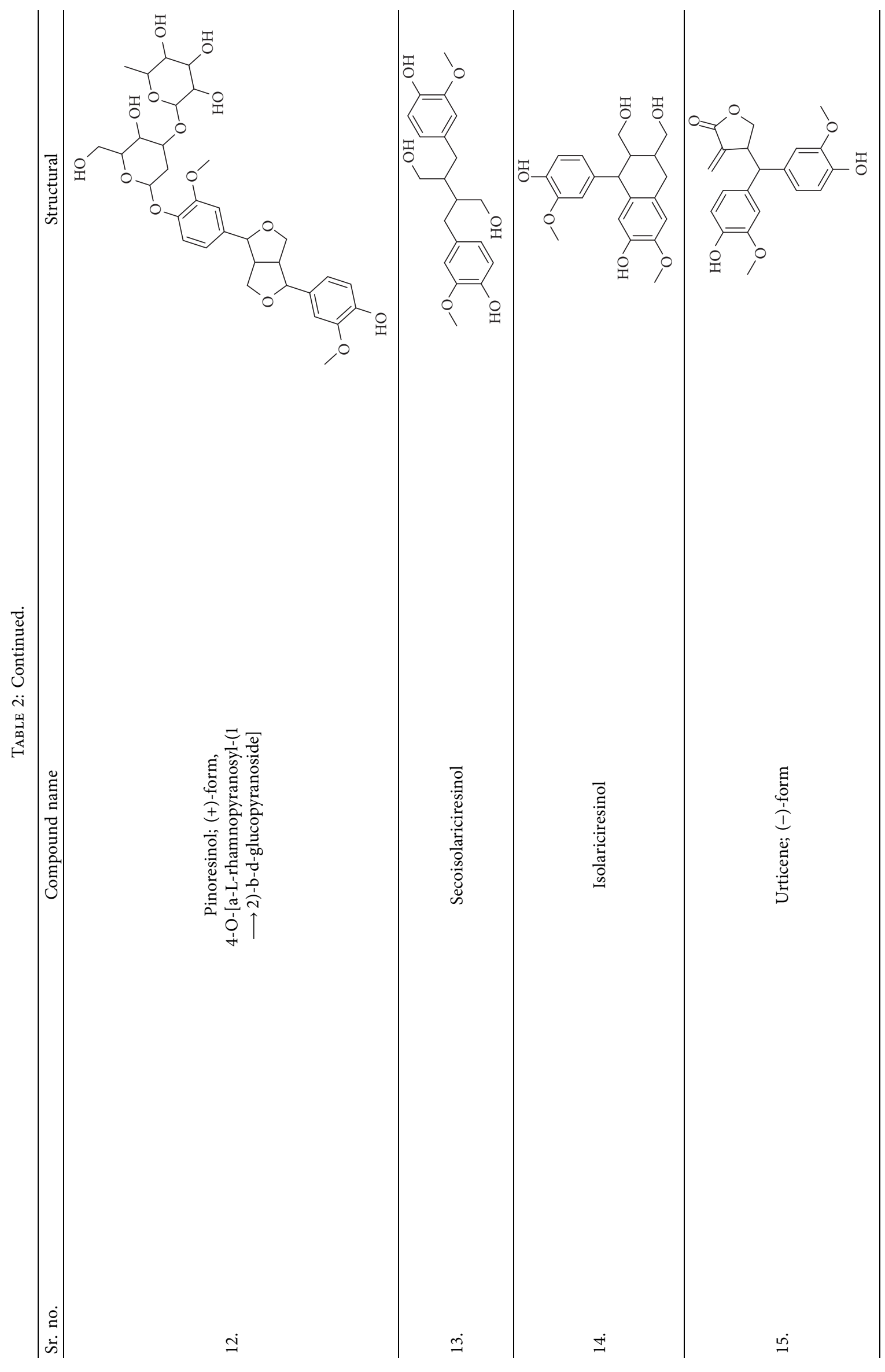




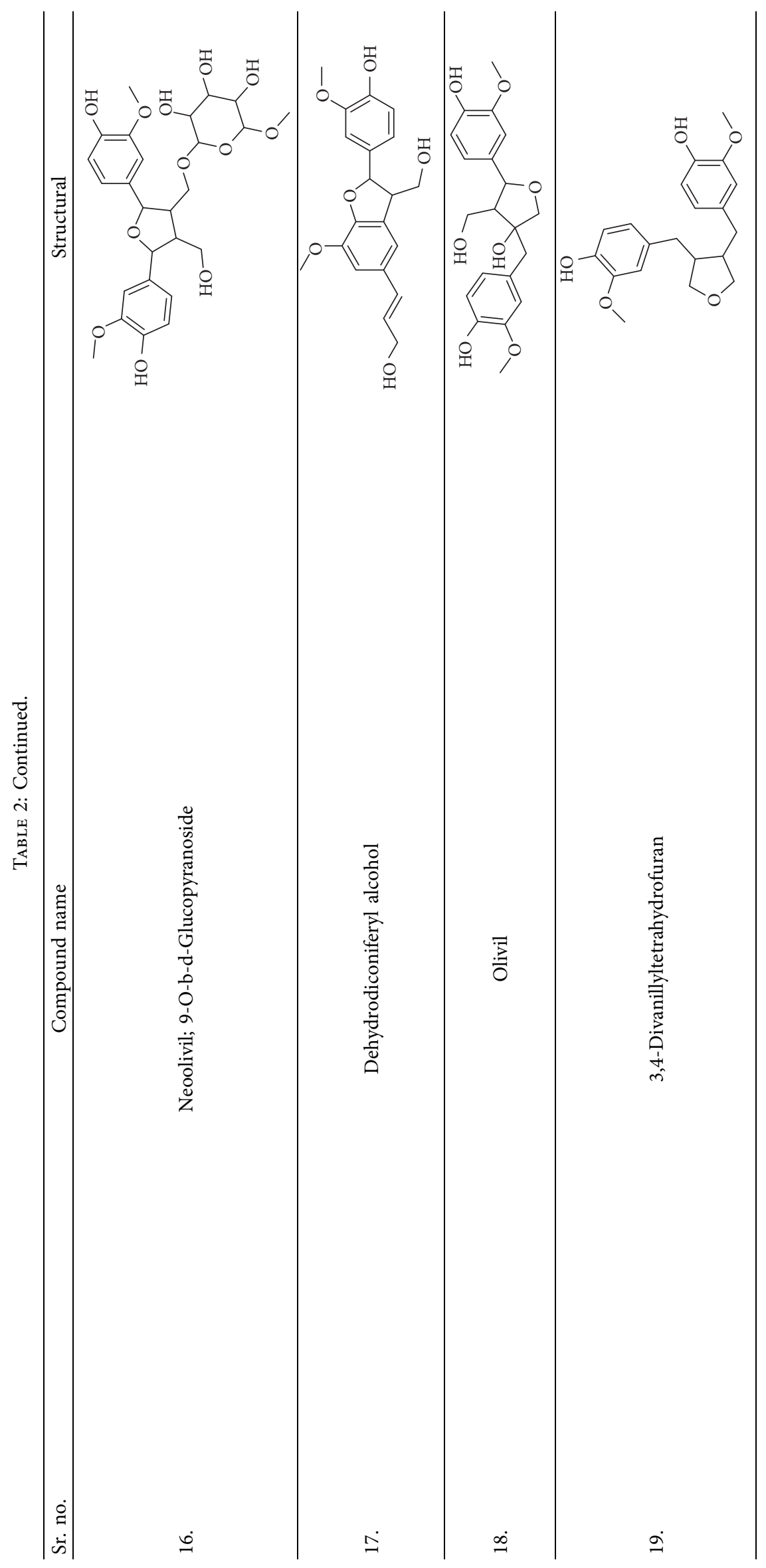


TABLE 3: Sterols extracted from Urtica.

\begin{tabular}{lcc}
\hline Sr. & Compound name & Structural formula \\
no. & Corm \\
\hline
\end{tabular}

1.

Stigmastane-3, 6-diol; (3 $\beta, 24 \mathrm{R})$-form, O-[b-d-Glucopyranosyl-(1 $\longrightarrow 4)$-alarabinopyranoside]

2. Stigmastane-3, 6-diol; (3b, 7a, 24R)-form, 3-O-b-d-Glucopyranoside

3.

Stigmastane-3, 6-diol; (3b, a6a, 24R)-form

4.

Daucosterol

5.

Ethyl iso-allocholate

6.

Cholesterol<smiles>CCC(CC)C(C)CCC(C)C1CCC2C3CC(O)=C4CC(OC5OCC(OC6OC(CO)C(O)C(O)C6O)C(O)C5O)CCC4(C)C3CCC12C</smiles><smiles>CCC(CCC(C)C1CCC2C3CC(O)=C4CC(OC5OCC(CO)C(O)C5O)CCC4(C)C3CCC12C)C(C)C</smiles><smiles>CCC(CCC(C)C1CCC2C3CC(O)C4CC(O)CCC4(C)C3CCC12C)C(C)C</smiles><smiles>CCC(CCC(C)C1CCC2C3CC=C4CC(OC5OC(CO)C(O)C(O)C5O)CCC4(C)C3CCC12C)C(C)C</smiles><smiles>CCOC(=O)CCC(C)C1CCC2C3C(O)CC4CC(O)CCC4(C)C3CC(O)C12C</smiles><smiles>CC(C)CCCC(C)C1CCC2C3CC=C4CC(O)CCC4(C)C3CCC12C</smiles>

Also, the N-acetyl glucosamine-specific lectin from Urtica dioica was inhibitory to cytomegalovirus (CMV), respiratory syncytial virus (RSV), and influenza A virus-induced cytopathic at an $\mathrm{EC}_{50}$ ranging from 0.3 to $9 \mathrm{mg} / \mathrm{ml} \mathrm{[74].}$
Another study showed that $U$. dioica agglutinin (UDA) suppressed the SARS-CoV virus replication by $90 \%$ at a concentration of $1.1 \pm 0.4 \mathrm{ug} / \mathrm{ml}$ in Vero 76 cells by likely targeting the early stages of the replication phase through 
TABle 4: Fatty acids isolated from genus Urtica.

Sr. Same Structural formula

TABle 5: Flavonoids isolated from genus Urtica.

\begin{tabular}{l} 
Sr. \\
\hline \\
1. \\
$2^{\prime}, 4^{\prime}, 5,7,8$-Pentahy-droxyflavone; 7, 8-Di-Me ether
\end{tabular}

2.

3.

4.

5.

6.

7.

8.

Luteolin 7-O-b-d-Glucopyranoside

Afzelin

Quercetin

Kaempferol

Gossypetin

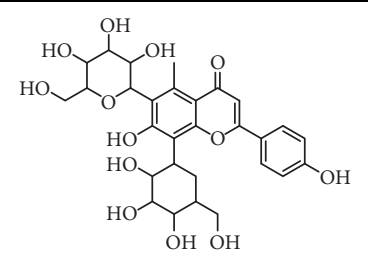

OH
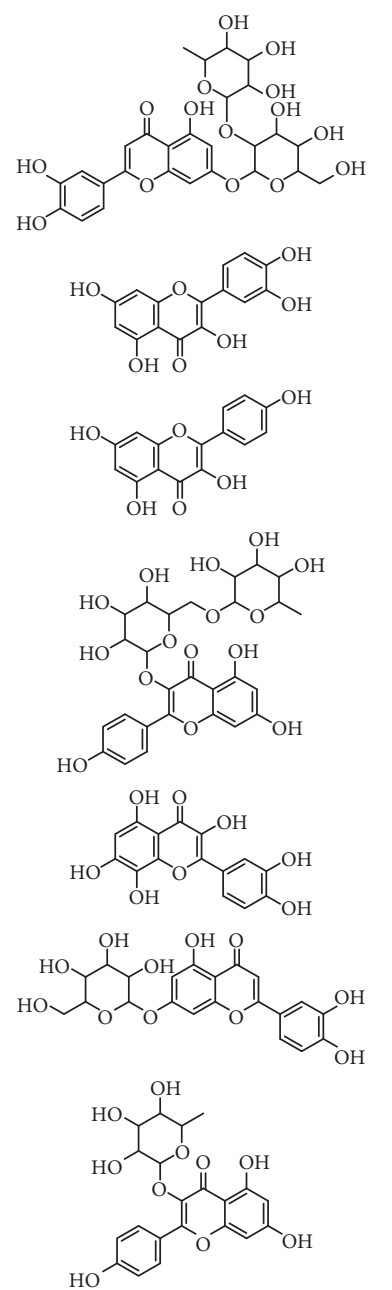
TABLE 5: Continued.

Sr.

TABle 6: Phenols extracted from Urtica spp.

Sr. no.

1.

2.

3.

4.

5.

6.

7.

\section{Compound name}

p-Coumaric acid

Vanillic acid

4-Methoxybenzoic acid

Ferulic Acid

Chlorogenic acid

Salicylic acid
Structural formula<smiles>O=C(O)/C=C/c1ccc(O)cc1</smiles><smiles>COc1cc(C(=O)O)ccc1O</smiles><smiles>COc1ccc(C(=O)O)cc1</smiles><smiles>O=C(O)CC(OC(=O)/C=C/c1ccc(O)c(O)c1)C(=O)O</smiles><smiles>COc1cc(/C=C/C(=O)O)ccc1O</smiles><smiles>O=C(/C=C/c1ccc(O)c(O)c1)OC1CC(O)(C(=O)O)CC(O)C1O</smiles><smiles>O=C(O)c1ccccc1O</smiles> 
TABle 6: Continued.

\begin{tabular}{lcl}
\hline Sr. no. & Compound name & Structural formula \\
8. & Caffeic acid &
\end{tabular}

TABLE 7: Alcohols isolated from genus Urtica.

Sr. Name

TABLE 8: Alkaloids isolated from genus Urtica.

Benzylisoquinoline


TABLE 8: Continued.

Name

TABle 9: Benzopyranoids isolated from genus Urtica.

\begin{tabular}{|c|c|c|}
\hline Sr. & Name & $\begin{array}{l}\text { Chemical } \\
\text { structure }\end{array}$ \\
\hline
\end{tabular}

1. $6,6^{\prime}, 7,7^{\prime}$-Tetrahy-droxy-[8, 8'-bi-2H-1-benzopyran]-2, 2'-dione; Tetra-Me ether

2.

7, $7^{\prime}$-dimethoxy-6, 6' -biscoumarin

3.

4.

$6,6^{\prime}, 7,7^{\prime}$-Tetrahydroxy-8, $8^{\prime}$-bicoumarin; 6, 6' -Di-Me ether

5.

6.

Scopoletin<smiles></smiles>

binding to the glycoprotein associated with the pseudotyped virus, thereby preventing the virus attachment to host cells [70].

4.1.2. Antimicrobial and Antifungal Activity. Despite the growing number of antimicrobials available, the rate of microorganisms with acquired drug resistance is alarming, and thus more research is needed to discover alternative therapies more effective and safer than the currently available ones $[75,76]$.

$U$. dioica ethanol and aqueous extracts showed antibacterial activity against both Gram-positive and Gram- 
TABle 10: Other compounds isolated from genus Urtica.

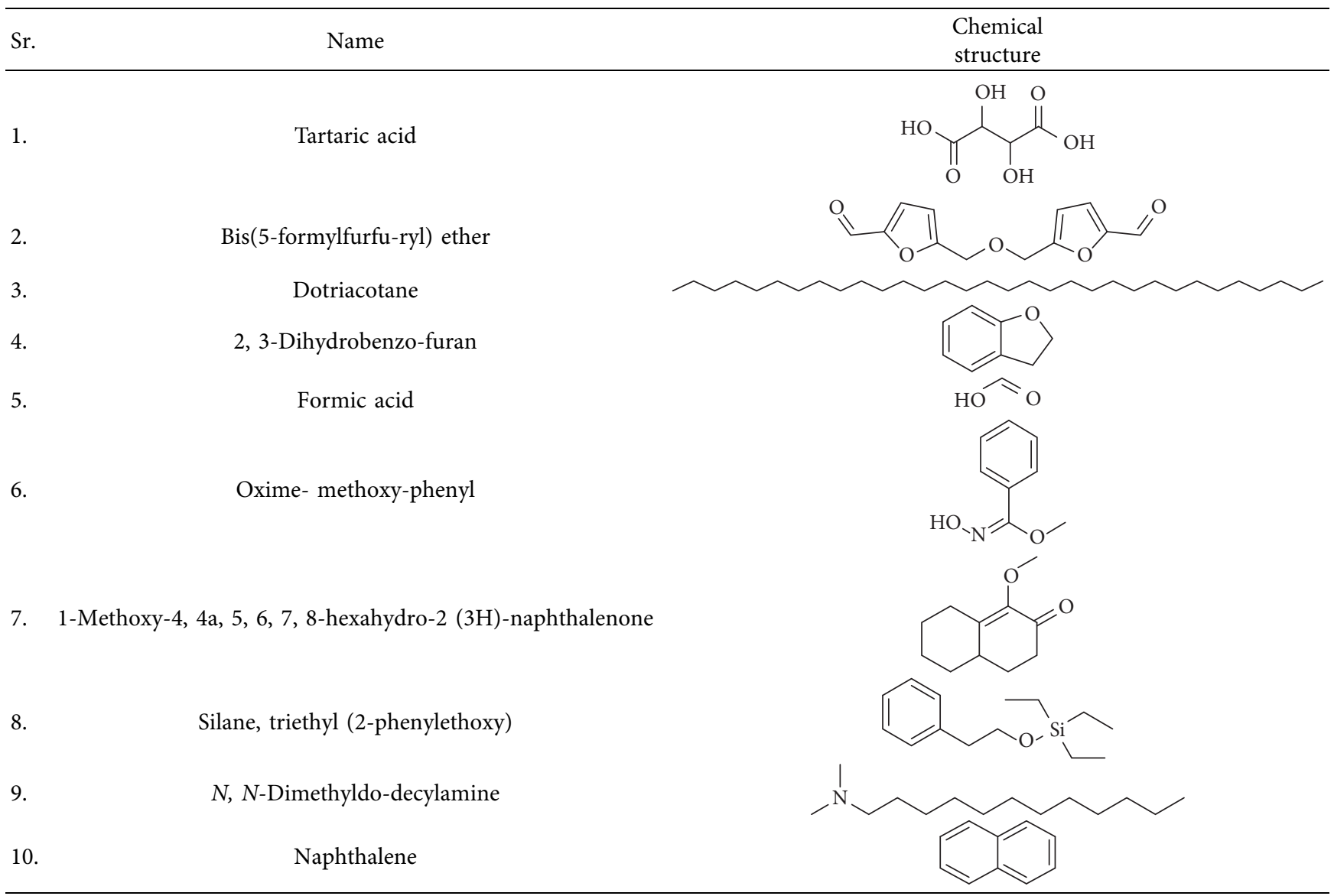

TABLE 11: Terpenoids isolated from genus Urtica.

\begin{tabular}{lcc}
\hline Sr. Name & $\begin{array}{c}\text { Structural } \\
\text { name }\end{array}$ \\
\hline
\end{tabular}

1.

$3^{\prime}$-Hydroxyacetophenone

2. 4,7-Megastigma-diene-3, 9-diol; (3S,6R,7E,9R)-form, 3-Ketone, 9-O[b-D-glucopyranosyl-(1 $\longrightarrow 2)$-b-d-glucopyranoside]

3. 1-(3, 4-Dihydroxyphe-nyl)-1, 2-propanediol; 3'-Me ether<smiles>CCCCCC(O)C=CC=CCCCCCCCC(=O)O</smiles>

4.

(9Z,11E)-1, 3-hy-droxy-9, 11-octadeca-dienoic acid 
TABle 11: Continued.

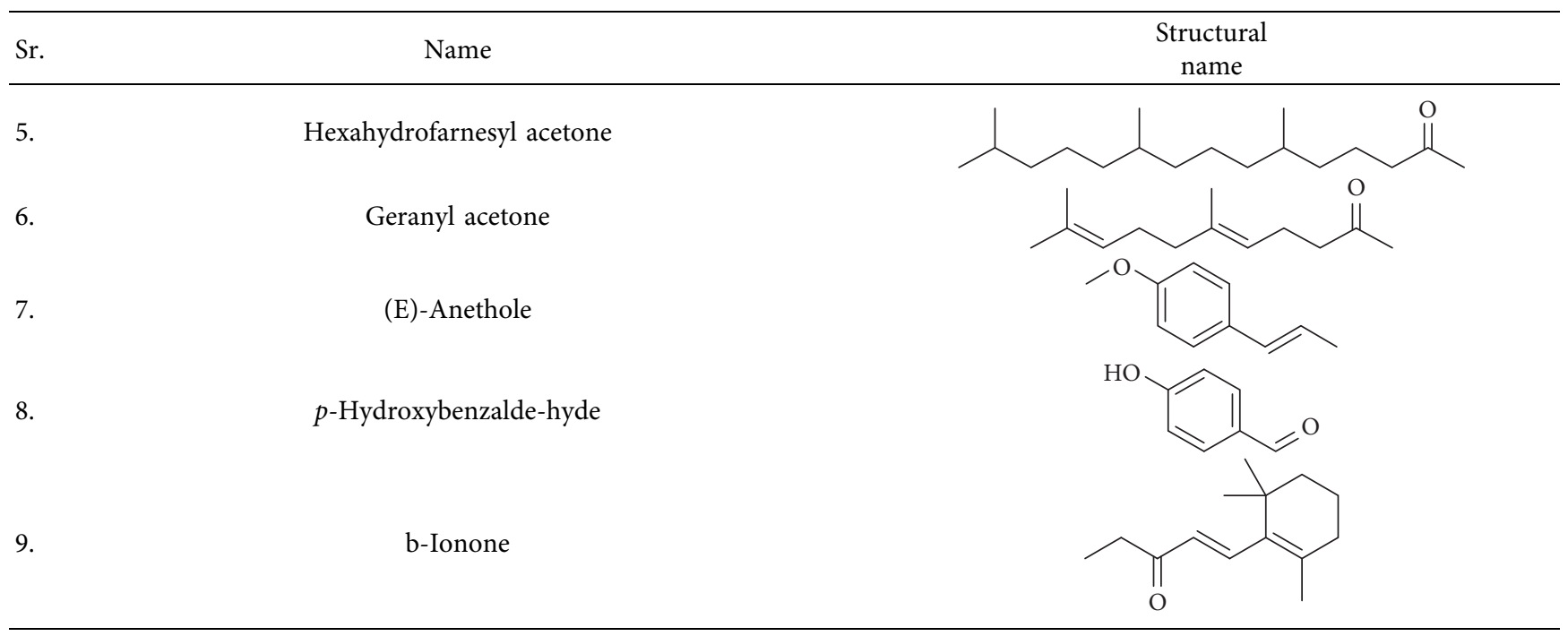

TABLE 12: Chemical composition of essential oil extracted of Urtica pilulifera and Urtica diorca.

\begin{tabular}{|c|c|c|c|c|}
\hline NO & Compound & Urtica pilulifera (RT) & Urtica diorca $(\mathrm{RI})$ & $\%$ \\
\hline 1 & 1-(4-Isopropylphenyl)-2-methylpropyl acetate & 30.5829 & - & 2.062 \\
\hline 2 & 1-(4'-pentenyl)-1, 2-epoxycyclopentane & 24.2355 & - & 0.1271 \\
\hline 3 & 1, 2-Benzenedicarboxylic acid & 32.0424 & - & 13.5056 \\
\hline 4 & 1, 4-Diazepine & 27.0528 & - & 0.3009 \\
\hline 5 & 1,8-Cineole & 13.686 & - & 8.2085 \\
\hline 6 & 1-Penten-3-one & 27.5551 & - & 0.3782 \\
\hline 7 & 2-(1-Pentenyl)furan & - & 1056 & 0.29 \\
\hline 8 & 2, 2, 6-Trimethylcyclohexanone & - & 1035 & 0.28 \\
\hline 9 & 2, 4, 6-Trimethyl-5H-1, 3, 5-dithiazine & - & 1199 & 0.30 \\
\hline 10 & 2-Methoxy-4-vinylphenol & 12.3214 & - & 0.1087 \\
\hline 11 & 2-Pentylfuran & - & 991 & 0.84 \\
\hline 12 & 2-Propenoic acid & 5.302 & - & 2.2418 \\
\hline 13 & 3, 5-Dimethyl-1, 2, 4-trithiolane & - & 1134 & 0.30 \\
\hline 14 & 3-Carene & 15.8651 & - & 3.7624 \\
\hline 15 & 3-Octanone & - & 988 & 0.28 \\
\hline 16 & 5, 6-Dihydro-4-pentyl-2, 6-dimethyl-4H-1, 3, 5-dithiazine & - & 1588 & 0.57 \\
\hline 17 & 5, 6-Dihydro-4-pentyl-2, 6-dimethyl- $4 \mathrm{H}-1,3,5$-dithiazine & - & 1588 & 0.57 \\
\hline 18 & 7-Acetyl-6-ethyl-1, 1, 4,4-tetramethyltetralin & 32.5855 & - & 19.618 \\
\hline 19 & .alpha-Cetone & 26.6658 & - & 0.9039 \\
\hline 20 & Anozol & 29.1437 & - & 0.1346 \\
\hline 21 & Apoatropine & - & 2093 & 0.82 \\
\hline 22 & Apoatropine & - & 2093 & 0.82 \\
\hline 23 & Benzaldehyde & 11.371 & - & 0.1391 \\
\hline 24 & Benzaldehyde & - & 964 & 0.29 \\
\hline 25 & Benzofuranone & 27.9014 & - & 0.1183 \\
\hline 26 & Benzoic acid & 30.7118 & - & 0.873 \\
\hline 27 & Bicyclo[10.1.0]trideca-4, 8-diene-13-carboxamide & 25.2741 & - & 0.1325 \\
\hline 28 & Bisabolene & - & 1506 & 0.39 \\
\hline 29 & Bisomel & 32.28 & - & 3.7872 \\
\hline 30 & Borneol & - & 1171 & 0.31 \\
\hline 31 & Bornyl acetate & - & 1283 & 2.14 \\
\hline 32 & Butyl acetate & 6.7208 & - & 3.2399 \\
\hline 33 & Cadinene & - & 1510 & 1.57 \\
\hline 34 & Cadinene & - & 1516 & 2.37 \\
\hline 35 & Camphor & - & 1145 & 0.27 \\
\hline 36 & Carvacrol & - & 1299 & 0.30 \\
\hline 37 & Carvone & 20.9362 & - & 0.1721 \\
\hline
\end{tabular}


TABle 12: Continued.

\begin{tabular}{|c|c|c|c|c|}
\hline $\mathrm{NO}$ & Compound & Urtica pilulifera (RT) & Urtica diorca $(\mathrm{RI})$ & $\%$ \\
\hline 38 & Citronellyl & 19.7143 & - & 0.1388 \\
\hline 39 & Copaene-8-ol & - & 1579 & 3.28 \\
\hline 40 & Decan-2-one & - & 1192 & 0.28 \\
\hline 41 & Decanal & - & 1206 & 0.29 \\
\hline 42 & Diethoxylated tridecyl alcohol & 30.9494 & - & 0.1828 \\
\hline 43 & Ethylhexyl benzoate & 31.187 & - & 0.3837 \\
\hline 44 & Farnesol & - & 1715 & 1.88 \\
\hline 45 & Farnesol & - & 1715 & 1.88 \\
\hline 46 & Farnesylacetone & - & 1908 & 1.26 \\
\hline 47 & Farnesylacetone & - & 1908 & 1.26 \\
\hline 48 & Furan-3-aldehyde & 7.325 & - & 0.1458 \\
\hline 49 & Geranyl acetone & 25.953 & - & 0.3483 \\
\hline 50 & Geranyl acetone & - & 1448 & 2.22 \\
\hline 51 & Geranyl acetone & - & 1448 & 2.22 \\
\hline 52 & Hexahydrofarnesylacetone & - & 1844 & 31.20 \\
\hline 53 & Hexahydrofarnesylacetone & - & 1844 & 31.20 \\
\hline 54 & Hexatriacontane & 6.7208 & - & 11.5631 \\
\hline 55 & Humulene & - & 1453 & 0.75 \\
\hline 56 & -Ionon $\beta$ & 26.822 & - & 0.1714 \\
\hline 57 & Ionone & - & 1421 & 4.04 \\
\hline 58 & Ionone & - & 1479 & 11.86 \\
\hline 59 & Isopropyl dodecanoate & - & 1627 & 5.27 \\
\hline 60 & Isopropyl dodecanoate & - & 1627 & 5.27 \\
\hline 61 & Lilyal & 27.7113 & - & 1.8666 \\
\hline 62 & Limonene (-)- & 13.5638 & - & 1.2463 \\
\hline 63 & Limonene (+)- & 23.3733 & - & 6.7658 \\
\hline 64 & Menthol & - & 1178 & 0.29 \\
\hline 65 & Methyl dihydrojasmonate & 30.3181 & - & 0.8451 \\
\hline 66 & Methyl palmitate & - & 1925 & 0.28 \\
\hline 67 & Methyl palmitate & - & 1925 & 0.28 \\
\hline 68 & Methylbenzene & 5.302 & - & 1.6415 \\
\hline 69 & Neophytadiene & 32.3751 & - & 5.2683 \\
\hline 70 & n-Nonanal & 15.9873 & - & 0.3288 \\
\hline 71 & n-Octanal & - & 1004 & 0.30 \\
\hline 72 & Nonanal & - & 1105 & 0.59 \\
\hline 73 & Ocimene & 20.59 & - & 0.6869 \\
\hline 74 & Octanal & 31.6555 & - & 2.0563 \\
\hline 75 & Octyl heptafluorobutyrate & 17.9696 & - & 0.1347 \\
\hline 76 & p-Guaiacol & 15.5392 & - & 0.1521 \\
\hline 77 & Phytol & - & 2110 & 11.20 \\
\hline 78 & Phytol & - & 2110 & 11.20 \\
\hline 79 & Safranal & - & 1196 & 0.33 \\
\hline 80 & $\beta$-Selinene & - & 1485 & 0.78 \\
\hline 81 & Terpinene & 14.5413 & - & 0.1705 \\
\hline 82 & Thymol & - & 1292 & 0.60 \\
\hline 83 & Trans-2,3-dimethylbicyclo[2.2.2]octane & 22.4161 & - & 0.3454 \\
\hline 84 & Vanillin & 21.812 & - & 1.7906 \\
\hline 85 & Vetivenene & - & 1532 & 0.49 \\
\hline 86 & Vinyl & 31.0445 & - & 0.3754 \\
\hline 87 & Xylene & 8.3229 & - & 0.3848 \\
\hline 88 & $\alpha$-Copaene-8-ol & - & 1579 & 3.28 \\
\hline 89 & $\alpha$-Humulene & - & 1453 & 0.75 \\
\hline 90 & $\alpha$-Ionone & - & 1421 & 4.04 \\
\hline 91 & $\alpha$-Longipinene & - & 1347 & 0.30 \\
\hline 92 & $\alpha$-Selinene & - & 1493 & 0.70 \\
\hline 93 & $\beta$-2--Pinene & 11.853 & - & 0.3957 \\
\hline 94 & $\beta$-Bisabolene & - & 1506 & 0.39 \\
\hline 95 & $\beta$-Caryophyllene & - & 1416 & 1.62 \\
\hline 96 & $\beta$-Cyclocitral & - & 1217 & 0.35 \\
\hline
\end{tabular}


TABle 12: Continued.

\begin{tabular}{lccc}
\hline NO & Compound & Urtica pilulifera $(\mathrm{RT})$ & Urtica diorca $(\mathrm{RI})$ \\
\hline 97 & $\beta$-Homocyclocitral & - & 1254 \\
98 & $\beta$-Ionone & - & 0.28 \\
99 & $\beta$-Selinene & - & 1479 \\
100 & $\beta$-Vetivenene & - & 1485 \\
101 & $\gamma$-Cadinene & - & 1.86 \\
102 & $\gamma$-Terpinen & 18.2615 & 0.78 \\
103 & $\gamma$-Terpinene & 18.6552 & 0.49 \\
104 & $\delta$-Cadinene & - & 1.57 \\
\hline
\end{tabular}

RI: retention time; RI: retention indices.

TABLE 13: In vivo studies of the genus Urtica.

\begin{tabular}{lllll}
\hline Extract/compound & Doses & $\begin{array}{c}\text { Route of } \\
\text { administration }\end{array}$ & Model & Effect \\
\hline
\end{tabular}

Antiarthritis effect

Total coumarins from Urtica dentata Hand

$20,40,60 \mathrm{mg} / \mathrm{kg}$

Orally every other day for 4 weeks after induction of arthritis
Collagen-induced arthritis $\mathrm{BALB} / \mathrm{c}$ mice model
Dose-dependent $\downarrow$ arthritis score $\downarrow$ paw swelling protect tissues against bone destruction $\downarrow$ IFN-g, $\downarrow$ IL-2 $\uparrow$ IL-10, $\uparrow$ TGF-B

Antioxidant effect

Total $80 \%$ ethanolic extract of Urtica dioica L. leaves
$50,100 \mathrm{mg} / \mathrm{kg}$

Orally daily for 14 Normal Swiss albino days mouse model $\uparrow$ cytochrome b5, $\uparrow \mathrm{NADH}-$ cytochrome b5 reductase, $\uparrow$ glutathione S-transferase, $\uparrow$ DT-diaphorase, $\uparrow$ glutathione peroxidase, $\uparrow$ glutathione reductase, $\uparrow$ superoxide dismutase, $\uparrow$ catalase $\downarrow$ cytochrome P450, \lactate

dehydrogenase, $\downarrow$ NADPHcytochrome $\mathrm{P} 450$ reductase, $\downarrow$ total sulfhydryl groups, $\downarrow$ nonprotein sulfhydryl groups, $\downarrow$ protein-bound sulfhydryl groups

Antidiabetic effect

Hypoglycemic effect - ethyl acetate and chloroform extracts $\downarrow$ glucose level, $\downarrow$ HbA1C, $\downarrow$ insulin resistance antiinflammatory: $\downarrow$ CRP, $\downarrow$ TNF- $\alpha$ antioxidant: $\downarrow \mathrm{MDA}, \uparrow \mathrm{GSH}, \uparrow \mathrm{SOD}$,
Orally daily for 4 chloroform extracts of Urtica pilulifera and $500 \mathrm{mg} / \mathrm{kg}$
Hexane, ethyl acetate and

Two doses: 250
Streptozotocin and type 2 diabetes adult male albino rat model high-fat diet-induced induction weeks starting from
day11 of diabetes
Intraperitoneally

daily for 16 days

Intraperitoneally
daily for 16 days
$\mathrm{ZnO}+$ extract: $8 \mathrm{mg} / \mathrm{dl}$

Alloxan-induced diabetic rat model nanoparticles + aqueous extract of Urtica dioica leaves

(a)

\section{$\uparrow$ catalase
Both $\mathrm{ZnO}$-extract and} insulin (reference) $\downarrow$ fasting blood glucose level in serum, while increased insulin level. $\mathrm{ZnO}$-extract: $\uparrow$ high-density lipoprotein $\downarrow$ total cholesterol, $\downarrow$ triglycerides

\section{Antiendometriosis effect}

Hexane, ethyl acetate and methanol extracts of Urtica dioica L. aerial parts
$100 \mathrm{mg} / \mathrm{kg} \quad$ Orally for 4 weeks

Surgery-induced endometriosis rat model
Methanol extract: $\downarrow$ implant volumes, \adhesion scores $\downarrow$ TNF- $\alpha$, \VEGF, $\downarrow$ IL-6; histopathological outcomes

[6

4]


TABle 13: Continued.

\begin{tabular}{lllll}
\hline Extract/compound & Doses & $\begin{array}{c}\text { Route of } \\
\text { administration }\end{array}$ & Model & Effect \\
\hline
\end{tabular}

Effect on prostate hyperplasia

\begin{tabular}{|c|c|c|c|c|c|}
\hline \multirow[b]{2}{*}{$\begin{array}{l}\text { Polysaccharide fraction of } \\
\text { Urtica fissa }\end{array}$} & & & & & \\
\hline & $\begin{array}{c}62.5,125,250 \mathrm{mg} / \\
\mathrm{kg}\end{array}$ & $\begin{array}{l}\text { Orally daily for } 3 \\
\text { weeks }\end{array}$ & $\begin{array}{l}\text { Testosterone } \\
\text { propionate-induced } \\
\text { prostate hyperplasia } \\
\text { castrated rat model }\end{array}$ & $\begin{array}{l}\downarrow \text { prostate hyperplasia the } \\
\text { lowest dose }(62.5 \mathrm{mg} / \mathrm{kg})-\downarrow \\
\text { indexes of wet weight, } \downarrow \text { dry } \\
\text { weight , } \downarrow \text { volume by } 17 \% \text {, } \\
23 \% \text { and } 32 \% \text { highest dose } \\
(250 \mathrm{mg} / \mathrm{kg})-\downarrow \text { indexes of wet } \\
\text { weight, dry weight, } \downarrow \text { volume } \\
\text { were further reduced by } \\
25 \%, 33 \% \text { and } 37 \% \text {; } \\
\text { histopathological } \\
\text { examination supported the } \\
\text { results }\end{array}$ & {$[68]$} \\
\hline \multicolumn{6}{|c|}{ Effect on nephrotoxicity } \\
\hline $\begin{array}{l}\text { Total } 95 \% \text { ethanol extract of } \\
\text { Urtica dioica }\end{array}$ & Dose: $100 \mathrm{mg} / \mathrm{kg}$ & $\begin{array}{l}\text { Orally daily for } 10 \\
\text { days }\end{array}$ & $\begin{array}{l}\text { Gentamicin-induced } \\
\text { nephrotoxicity in } \\
\text { male rabbit model }\end{array}$ & $\begin{array}{c}\downarrow \text { serum creatinine, } \downarrow \text { blood } \\
\text { urea, } \downarrow \text { nitrogen } \\
\text { antioxidant- } \uparrow \text { glutathione, } \\
\downarrow \text { malondialdehyde }\end{array}$ & [69] \\
\hline
\end{tabular}

Antiviral effect

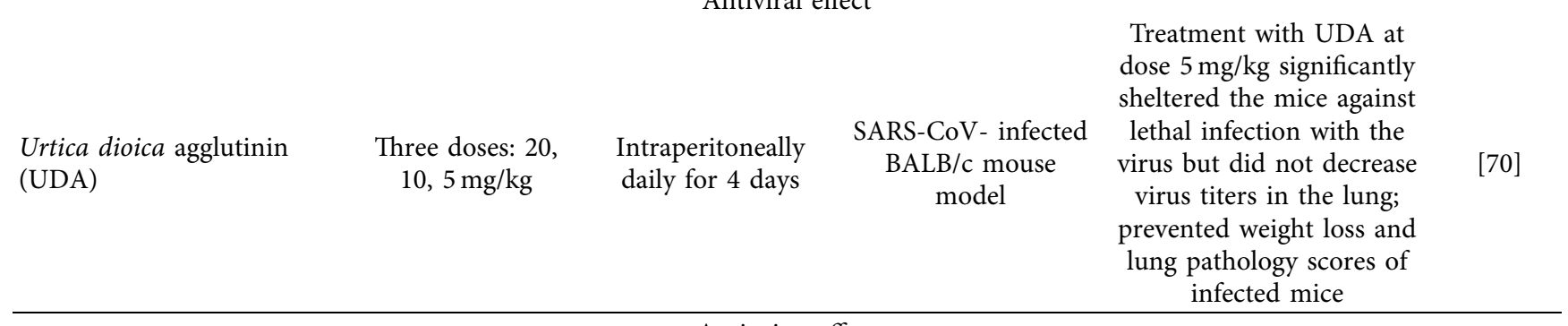

Antiaging effect

\begin{tabular}{|c|c|c|c|c|c|}
\hline $\begin{array}{l}\text { Total extract ( } 50 \% \text { ethanol) } \\
\text { of Urtica thunbergiana leaves }\end{array}$ & $\begin{array}{l}\text { Two doses: } 0.1 \% \\
\text { and } 1 \% \text { g/kg of the } \\
\text { animals' dry diet }\end{array}$ & Orally for 10 weeks & $\begin{array}{l}\text { UVB-induced skin } \\
\text { aging hairless mouse } \\
\text { model }\end{array}$ & $\begin{array}{c}\downarrow \text { thinner and superficial } \\
\text { wrinkles } \downarrow \text { erythema index } \\
\uparrow \text { skin hydration; } \\
\text { histopathological } \\
\text { investigations supported the } \\
\text { results }\end{array}$ & {$[71]$} \\
\hline
\end{tabular}

Anticancer effect

\begin{tabular}{|c|c|c|c|c|c|}
\hline & & Anticance & & & \\
\hline $\begin{array}{l}\text { Dichloromethane extract of } \\
\text { Urtica dioica }\end{array}$ & $\begin{array}{l}\text { Two doses: } 10 \text { and } \\
20 \mathrm{mg} / \mathrm{kg}\end{array}$ & $\begin{array}{l}\text { Intraperitoneally } \\
\text { daily for } 28 \text { days }\end{array}$ & $\begin{array}{c}\text { 4T1 (breast cancer } \\
\text { cell line) allograft } \\
\text { tumor BALB/c } \\
\text { mouse model }\end{array}$ & $\begin{array}{c}\text { tumor size and weight. } \\
\uparrow \text { apoptosis, } \downarrow \text { proliferation. } \\
\downarrow \text { Bcl } 2, \uparrow \text { caspase } 3 \text {; } \\
\text { histopathology } \\
\text { examinations supported the } \\
\text { results }\end{array}$ & [72] \\
\hline
\end{tabular}

Symbols: $\uparrow$ increase, $\downarrow$ decrease.

negative bacteria and yeasts, including Proteus mirabilis, Pseudomonas aeruginosa, Enterobacter aerogenes, Escherichia coli, Citrobacter koseri, S. pneumonia, S. aureus, M. luteus, S. epidermidis, and Candida albicans. They were also active against $M$. tuberculosis in case of multiple drug resistance [77-79]. Of note, the aqueous (microwaveassisted, ultrasound-assisted, and subcritical water extraction) and ethanol extracts of $U$. dioica leaves also confirmed antibacterial activity with minimal inhibitory concentration (MIC) of $9.76 \mathrm{ug} / \mathrm{mL}$ and $0.0625-0.500 \mathrm{mg} /$ $\mathrm{ml}$ against methicillin-resistant (MRSA) and methicillin- sensitive (MSSA) S. aureus strains [80]; these observed effects were linked to their high content of hydroxycinnamic acids (chlorogenic, caffeic, and rosmarinic acids) and flavonoids (quercetin) (Table 1) [81].

4.1.3. Anthelmintic Activity. The ethanolic extract of $U$. dioica displayed in vitro anthelmintic activity against protoscoleces of Echinococcus granulosus, increasing the concentration and duration of exposure, reaching $96.2 \%$ inhibition at a concentration of $4 \mu \mathrm{g} / \mathrm{ml}$ for $30 \mathrm{~min}$ (Table 1) 


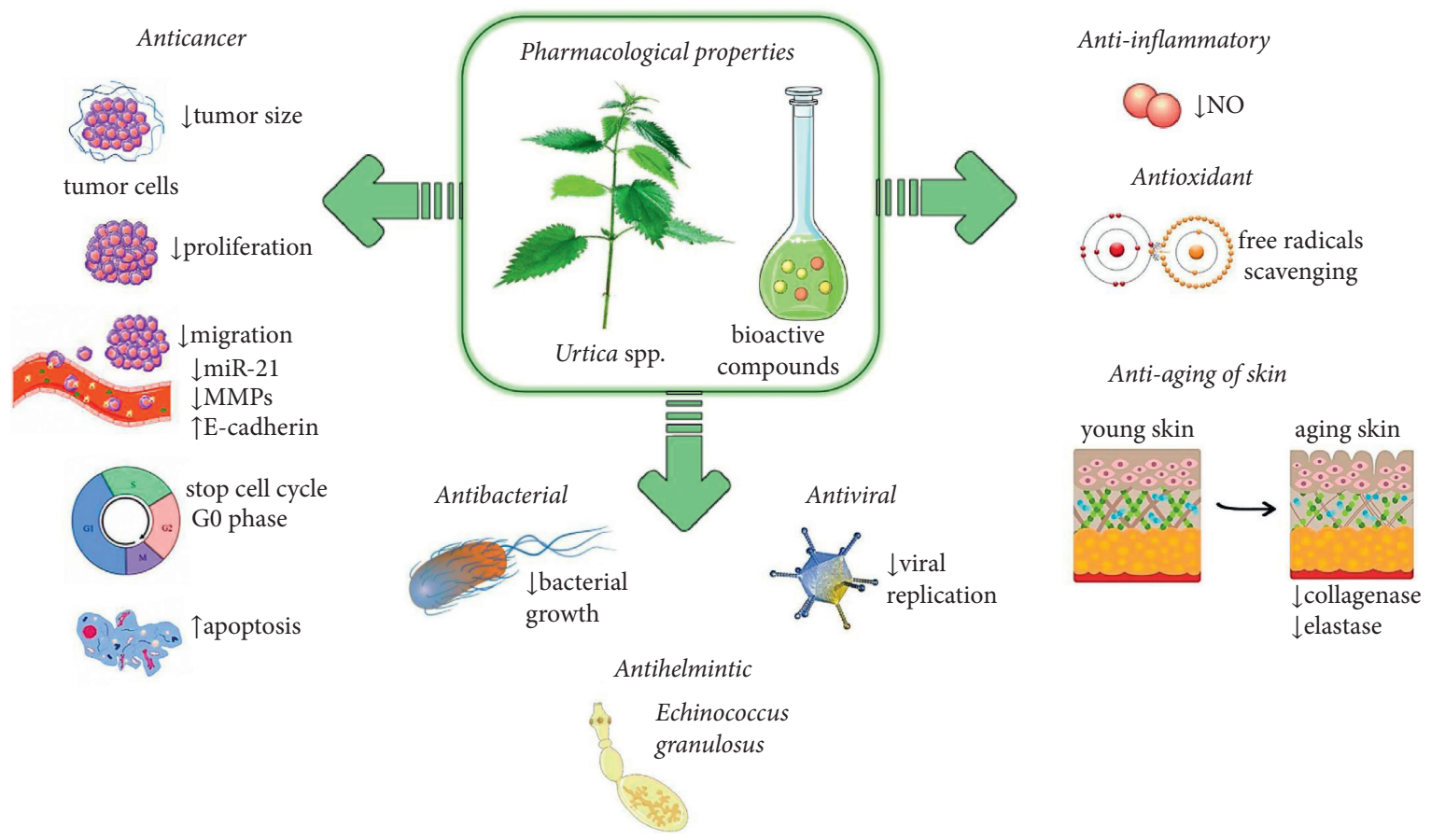

FIgURE 1: The most important pharmacological properties and potential mechanisms of bioactive compounds of Urtica spp. $\uparrow:$ increase; $\downarrow$ : decrease; NO: nitric oxide; MMPs: matrix metalloproteinases; miR-21: microRNA-21.

[82]. Anthelmintic activity of the methanol extract was also investigated using adult Indian earthworms (Pheretima posthuma) and revealed a dose-dependent increase in anthelmintic activity at 25,50 , and $100 \mathrm{mg} / \mathrm{mL}$ [83].

4.1.4. Anticancer Activity. Cancer is the largest cause of death in the world due to poor timely access to high-quality diagnosis and treatment [84, 85]. U. dioica significantly suppressed the human breast cancer cell line (MCF-7) and fibroblasts secluded from foreskin tissue, with $\mathrm{IC}_{50}$ values of MCF-7 $(31.37 \mathrm{mg} / \mathrm{ml})$, MDA-MB-23 $(38.14 \mathrm{mg} / \mathrm{ml}), 4 \mathrm{~T} 1$ $(44.07 \mu \mathrm{g} / \mathrm{mL}$ to $35.21 \mathrm{mg} / \mathrm{ml})$, and HFFF2 $(69.42 \mathrm{mg} / \mathrm{ml})$. QRT-PCR showed that $U$. dioica extracts inhibited cell migration by downregulating the expression of miR-21, matrix metalloproteinase (MMP) 1, MMP9, and MMP13, and $\mathrm{C}-\mathrm{X}-\mathrm{C}$ motif chemokine receptor 4 (CXCR4) and upregulating the expression of E-cadherin [86]. U. dioica leaves also increased cell apoptosis in $4 \mathrm{~T} 1$ cells [72].

The aqueous extract of $U$. dioica leaves significantly decreased the cell proliferation of AML U937 cell line (acute myeloid leukemia), with $\mathrm{IC}_{50}$ of $24 \mathrm{ug} / \mathrm{ml}$ for the first $48 \mathrm{~h}$ and then $16 \mathrm{ug} / \mathrm{ml}$ after $72 \mathrm{~h}$ [87]. Moreover, flow cytometry showed that the extract was able to stop the cell cycle into the G0 phase and increase cell apoptosis at the early and late stages by increasing proapoptotic protein Bax expression and decreasing antiapoptotic protein $\mathrm{Bcl}-2$ expression [87].

Zekovic et al. reported the antiproliferative effect of the subcritical water extract of $U$. dioica against Hep2c, $\mathrm{RD}$, and L2OB cells $(13.42 \mathrm{ug} / \mathrm{ml}, 9.69 \mathrm{ug} / \mathrm{ml}$, and $7.52 \mathrm{ug} / \mathrm{ml}$, respectively) (Table 1) [80]. Moreover, the bioactive compound, $5 \mathrm{a}$, 6b-dihydroxy-daucosterol, from U. laetevirens showed anticancer activity against MH7A cells by inhibiting proliferation and inducing apoptosis (Table 1) $[88,89]$.

4.1.5. Antioxidant Activity. Antioxidants are synthetic or natural compounds that can help to prevent or delay cell damage $[90,91]$. The aqueous extract of $U$. dioica leaves presented antioxidant activity, assessed through the DPPH radical scavenging $\left(\mathrm{IC}_{50}=16.93 \mathrm{ug} / \mathrm{mL}\right)$, reducing power $\left(\mathrm{EC}_{50}=30.07 \mathrm{ug} / \mathrm{mL}\right)$ and polarographic $(\mathrm{HPMC}=243.2 \% /$ $\mathrm{mL}$ ) assays [80].

Batches of $U$. dioica analyzed for their antioxidant potency revealed batch 14 as the most potent (2.71 TEAC) using the CUPRAC assay and batch 27 (0.73 TEAC) using the FRAP assay. The resulting response surface plots approved a positive association between the antioxidant actions and the phenolic acids content [92]. A comparative study performed by Carvalho et al. demonstrated the superior antioxidant properties of $U$. dioica in all assays: DPPH $(2.89 \mathrm{~g} / 100 \mathrm{~g}$ lyophilized $)$, ABTS (2.60 TEAC), and FRAP (3.81 TEAC) when compared to U. membranacea and U. urens aerial parts (Table 1) [89].

Methanol and direct-ethanol extracts of Urtica root showed free radical scavenging activity of $46.71 \%$ and $45.03 \%$ at $500 \mu \mathrm{g} / \mathrm{ml}$, respectively. Moreover, Urtica parviflora (methanol/aqueous extract) has been reported for free radical scavenging and reducing activity, with biological activity varying in a dose-dependent manner. The antioxidant potential has also been reported in the ethanolic extracts of Urtica circularis, Urtica hyperborean (methanol extract), Urtica cannabina (polyphenols), and U. urens (Table 1) [93-96]. 
4.1.6. Anti-Inflammatory Activity. Although nonsteroidal medicines can be useful, herbs can be a safer and often effective alternative for pain management, especially when used for a long period [97]. U. dioica (leaves extract) and isolated flavonoids were active against thrombin-induced platelet aggregation $\left(\mathrm{IC}_{50}\right.$ values of $0.25 \pm 0.05$ and $0.40 \pm 0.04 \mathrm{mg} / \mathrm{ml}$ ) [98]. A comparative study between $50 \%$ ethanol extracts of $U$. dioica, $U$. membranacea, and $U$. urens aerial parts showed that $U$. urens extract $(350 \mathrm{ug} / \mathrm{mL})$ could act as a more potent anti-inflammatory agent by showing the highest reduction in nitric oxide production (up to $41 \%$ ) (Table 1) [89].

4.1.7. Antiaging of Skin. Those who are prone to wrinkles and fine lines and those who have loose, sagging skin usually consider antiaging therapies [99]. Different extracts of $U$. dioica demonstrated antiaging efficacy using elastase and collagenase enzymes inhibition assay. The more potent batches were batch 1 that inhibited collagenase enzyme by $16.23 \%$ and batch 26 that inhibited elastase enzyme by $24.51 \%$. This potency was linked to the high content of quercetin and ursolic acid, respectively (Table 1) [92].

\subsection{In Vivo Pharmacological Findings}

4.2.1. Antiviral Activity. U. diorca was also investigated for its in vivo antiviral potency. $U$. dioica agglutinin (UDA) at a dose of $5 \mathrm{mg} / \mathrm{kg}$ (b.w/day; i.p.) significantly sheltered the mice against lethal infection with the virus but did not decrease the virus titers in the lung of the SARS-CoV-infected BALB/c mouse model, also preventing the weight loss and lung pathology scores of infected mice [70] (Table 13).

4.2.2. Anthelmintic Activity. The fight against helminth infectious is still pending complete eradication either through a vaccine or pharmacological therapies. In vivo study showed that daily oral administration $(175 \mathrm{mg} / \mathrm{ml})$ of the methanol extract obtained from leaves and seeds of $U$. dioica showed anthelmintic activity in Swiss albino mice naturally infected with Aspiculuris tetraptera (Table 13) [100].

4.2.3. Anticancer Activity. The dichloromethane extract of $U$. dioica further showed anticancer activity by significantly reducing the tumor size and weight on 4T1 (breast cancer cell line) allograft tumor in BALB/c mouse model at 10 and $20 \mathrm{mg} / \mathrm{kg}$ b.w/day (i.p.). This efficacy was linked to increased cell apoptosis and suppression of cell proliferation through BCL2 downregulation and increased caspase-3 activity [72].

4.2.4. Nephroprotective. The kidney is a key organ of the metabolism of any xenobiotic; thus, preventing its alteration is crucial [101]. The $95 \%$ ethanol extract of $U$. dioica showed therapeutic action against nephrotoxicity on gentamicininduced nephrotoxicity in the male rabbit model at a dose (100 mg/kg b.wt./day P.O.). The extract has a potent antioxidant activity through enhancing glutathione level and decreasing malondialdehyde level and helps in controlling serum creatinine and blood urea nitrogen levels [69]. Urtica parviflora extract (aerial parts) showed neuroprotective activity against nephrotoxicity induced by paracetamol and gentamicin and renal disability in Wistar rats and rabbits (Table 1) [77, 96].

4.2.5. Hepatoprotective. Because the liver is such an important part of any xenobiotic metabolism, preventing its alteration is also of utmost importance [102]. U. urens and $U$. dioica have been reported for their hepatoprotective activity against $\mathrm{CCl} 4$-induced liver toxicity in rats. For example, $U$. dioica (methanol extract) promoted an antioxidant system against cisplatin-induced toxicity in Ehrlich ascites tumor (mice model) and exerted hepatoprotective activity (Table 13) [83, 89].

4.2.6. Cardioprotective. Cardioprotection refers to all systems and methods that help keep the heart healthy by decreasing or even preventing myocardial damage [103]. $U$. dioica water and petroleum ether extract at, respectively, 20 and $150 \mathrm{mg} / \mathrm{kg} /$ day improved blood lipid level in rats, decreased blood cholesterol levels and LDL/HDL lipoprotein ratios after 30 days. On the other hand, $U$. dioica ethanol extract decreased cholesterol and LDL levels at a dose of 100 and $300 \mathrm{mg} / \mathrm{kg}[104,105]$. Urtica parviflora (350 and $500 \mathrm{mg} / \mathrm{kg}$ p.o.) effectively decreased cardiac complications and enhanced serum LDL level.

$U$. dioica aqueous extract ( 1 and $2 \mathrm{~g} / \mathrm{L}$ ) decreased heart rate and improved pressure in the left ventricle in Langendorff-perfused rat heart. It also improved the tolerance level of isolated rat heart against ischemia-reperfusion (Table 13) [106-108].

4.2.7. Antiarthritis Effect. Urtica species has also been shown to be effective for anti-inflammatory purposes, particularly in the treatment of arthritis. For example, a total coumarins extract from Urtica dentata demonstrated a dosedependent antiarthritis activity in collagen-induced arthritis BALB /c mice model at three doses $(20,40$, and $60 \mathrm{mg} / \mathrm{kg}$ b.w. P.O. every other day). Total coumarins also protected tissues against bone destruction by reducing IFN-g and IL-2 production and increasing IL-10 and TGF-B (Table 13) [63].

4.2.8. Antidiabetic Effect. Diabetes mellitus is a significant metabolic illness that can affect the central nervous system in a variety of ways, both functionally and morphologically [109]. Ethyl acetate and chloroform extracts of $U$. pilulifera showed antidiabetic activity at two doses (250 and $500 \mathrm{mg} /$ kg b.w./day P.O.) on streptozotocin and high-fat diet-induced type 2 diabetes adult male albino rat model. Briefly, the extracts decreased glucose level, HbA1C percentage, and insulin resistance, with this hypoglycemic effect being associated with the anti-inflammatory effect through reducing C-reactive protein (CRP) levels in serum and TNF-a level and exerting antioxidant activity through decreasing MDA and increasing GSH levels, SOD, and catalase 
activities in pancreatic tissues (Table 13) [65]. Also, a formulation containing $U$. dioica, Artemisia judaica, Morus folium, Taraxacum officinale, and Canella winteriana has been reported to treat insulin-dependent (type I) and noninsulin-dependent (type II) diabetes. Furthermore, a lectin isolated from seeds of $U$. pilulifera exerted an antidiabetic impact on diabetic rats (streptozotocin (STZ) model) when administered for 30 days at a dose of $100 \mathrm{mg} /$ kg. U. parviflora leaves (aqueous extract) also exerted hypoglycemic effect in normoglycemic rats, while $U$. angustifolia (leaves, stems, and roots) exerted hypoglycemic effects in a dose-dependent way (Table 13) [79, 110-112]. More recently, $\mathrm{ZnO}$ nanoparticles of aqueous extract from $U$. dioica leaves confirmed the antidiabetic activity of the combination $(8 \mathrm{mg} / \mathrm{dl}$. b.w./day I.P.) in an alloxan-induced diabetic rat model by significantly decreasing fasting blood glucose, total cholesterol, and total triglycerides levels in serum, while increasing high-density lipoprotein and insulin levels (Table 13) [66].

4.2.9. Antiendometriosis Effect. Endometriosis is a painful disorder in which tissue from the womb's lining (uterus) is present both inside and outside the uterus. Some herbs may raise the risk of endometriosis, while others may help to heal it faster. The methanol extract of $U$. dioica aerial parts showed an antiendometriosis effect on the surgery-induced endometriosis rat model at a dose of $100 \mathrm{mg} / \mathrm{kg}$ b.wt./day P.O. by decreasing implant volumes and adhesion scores and peritoneal TNF- $\alpha$, VEGF, and IL-6 levels as supported by histopathological outcomes (Table 13) [67].

4.2.10. Effect on Prostate Hyperplasia. Prostate enlargement, commonly known as benign prostatic hyperplasia (BPH), is a noncancerous increase in the size of the prostate gland. Prostatic hyperplasia was suppressed by a polysaccharide fraction of Urtica on testosterone propionate-induced prostate hyperplasia castrated rat model at three doses (62.5, 125 , and $250 \mathrm{mg} / \mathrm{kg}$ b.wt. P.O.). Treatment with the lowest dose $(62.5 \mathrm{mg} / \mathrm{kg})$ reduced the indexes of wet weight, dry weight, and volume by $17 \%, 23 \%$, and $32 \%$, respectively. With the highest dose $(250 \mathrm{mg} / \mathrm{kg})$, the indexes of wet weight, dry weight, and volume were further reduced by $25 \%, 33 \%$, and $37 \%$, respectively (Table 13) [68].

Many herbal preparations from $U$. dioica extracts can inhibit 5- $\alpha$-reductase [113]. Indeed, $U$. dioica roots (methanol extracts) were able to inhibit aromatase (AR) and 5areductase $(5 \mathrm{aRE})$ in a dose-dependent manner $\left(\mathrm{ED}_{50}\right.$ of 3.58 and $14.7 \mathrm{mg} / \mathrm{mL}$, respectively). Urtica mairei (roots) reduced BPH and inhibited the activity of 5a-reductase (Table 13) $[114,115]$.

Hartmann et al. evaluated the effect of a combination between methylene chloride extract of Pygeum africanum bark and 30\% methanol extract of $U$. dioica roots with a ratio of $1: 12$ (Prostatonin ${ }^{\circledR}$ ) on BPH. This combination also significantly inhibited reductase and aromatase enzymes with $\mathrm{ED}_{50}$ of $14.15 \mathrm{mg} / \mathrm{ml}$ and $0.24 \mathrm{mg} / \mathrm{ml}$, respectively (Table 13) [116].
4.2.11. Antioxidant Activity. Antioxidants are widespread in the plant kingdom. For example, the $80 \%$ ethanol extract of $U$. dioica leaves confirmed antioxidant activity in a normal Swiss albino mouse model at two doses $(50$ and $100 \mathrm{mg} / \mathrm{kg}$ b.w./day P.O.). Both doses of the extract led to a marked increase in the activities of cytochrome b5, NADH-cytochrome b5 reductase, glutathione S-transferase, DT-diaphorase, glutathione peroxidase, glutathione reductase, superoxide dismutase, and catalase in liver tissues. On the other hand, they showed a reduction in cytochrome P450, lactate dehydrogenase, NADPH-cytochrome P450 reductase, total sulfhydryl groups, nonprotein sulfhydryl groups, and protein-bound sulfhydryl groups (Table 13) [64].

4.2.12. Anti-Inflammatory Activity. The discovery of new anti-inflammatory agents has long been a source of concern. The aqueous extract of $U$. dioica leaves showed analgesic effect at $1200 \mathrm{mg} / \mathrm{kg}$ by reducing thermal situation in a hot plate test $\left(55^{\circ} \mathrm{C}\right)$, improving resistance to ache and hyperstimulation of the sensory nociceptors leading to TENS-like effect $[94,117]$.

The aerial part of $U$. urens (ethanol extract) inhibited $62.8 \%$ of the licking time during the final stage of the formalin test at a dose of $500 \mathrm{mg} / \mathrm{kg}$ in chemically induced mouse pain models $[118,119]$. U. urens (methanol extract of aerial parts) at 100 to $400 \mathrm{mg} / \mathrm{kg}$ significantly displayed anxiolytic effect against mice model (Table 1) [120].

Furthermore, $U$. dioica aqueous extract $(150 \mathrm{mg} / \mathrm{kg}$ dose) showed antipyretic activity in albino mice, while Urtica macrorrhiza aqueous extract (stem) decreased fever intensity in rats at 200 and $400 \mathrm{mg} / \mathrm{kg}[79,121]$. Indeed, it has been reported that $U$. dioica act by either blocking or interfering with chemical processes in the body related to chemicals found in the body, including dihydrotestosterone. In the carrageenan-induced paw edema model of rats, $U$. urens showed outstanding anti-inflammatory efficacy. Extract of its aerial parts revealed a percentage inhibition of $41.5 \%$ at $300 \mathrm{mg} / \mathrm{kg}$ i.p. in case of hind paw edema in rats. Moreover, petroleum ether extract of seeds of $U$. pilulifera and n-butanol and aqueous of $U$. macrorrhiza have also reported antiinflammatory activity against carrageenan-induced paw edema in rats (Table 13) $[111,120,122,123]$. In addition, some compounds from Urtica circularis, namely vicenin-2, caffeic acid, chlorogenic acid, and vitexin displayed a dosedependent antinociceptive activity in nociceptive mice, in the following order of activity: vitexin $(91 \%)>$ caffeic acid $(41 \%)=$ vicenin $-2(41 \%)>$ chlorogenic acid $(72 \%)($ Table 13$)$ $[118,119]$.

4.2.13. Antiaging of Skin. The use of natural plant extracts in the cosmetic industry as antiaging agents has received rising attention. Hwang et al. demonstrated the antiaging activity of the $50 \%$ ethanol extract of Urtica thunbergiana leaves on UVB-induced skin aging hairless mouse model at two doses $(0.1 \%$ and $1 \% \mathrm{~g} / \mathrm{kg}$ b.w. of animals' diet). The extract (100 ug/ $\mathrm{mL}$ ) improved the aging disorders implied by UVB-irradiated NHDF, with ROS generation being reduced by $17 \%$, MMP- 1 and MMP-3 by $61 \%$ and 29\%, respectively, and IL- 6 
secretion by $60 \%$. Moreover, procollagen type 1 generation was upregulated by $255 \%$ and phosphorylation of ERK, JNK, and $\mathrm{p} 38 \mathrm{~K}$ was suppressed by $14 \%, 32 \%$, and $38 \%$, respectively. Dephosphorylation of NFAT was also inverted possibly due to the high content of chlorogenic acid in U. thunbergiana (Table 13) [71].

4.2.14. Diuretic and Antiurolithiatic Effects. U. dioica has traditionally been used as a diuretic in indigenous medicine. Experimentally, $U$. dioica (aqueous extract) possess natriuretic and diuretic activity in rabbits; the rate of $\mathrm{K}^{+}$remains unaffected. $U$. dioica also revealed effectiveness against urinary infections. Indeed, its aerial part (methanol extract) also exerts antiurolithiatic potential can suppress the increased levels of urinary calcium and creatinine while significantly reducing the renal deposition of calcium and oxalate. $U$. dentata (n-butanol extract) also exert antiurolithiatic activity, prevent the deposition of calcium oxalate, and protect renal tissue from injury produced by kidney calculi (rat model) (Table 13) [112, 124].

4.3. Miscellaneous. The hypotensive activity of the methanol and water extract of $U$. dioica has also been shown in human cells culture and in vitro models of prostatic antihyperplasic activity [125]. U. dioica aqueous extract has been revealed to exert good in vivo antiulcer efficacy against ethanol-induced ulcers [113], while leaves and seed extract $(400 \mu \mathrm{g} / \mathrm{mL})$ possess in vitro immunomodulatory potential (Table 13) $[47,126]$. Finally, U. angustifolia (polysaccharides) showed antifatigue properties in mice [112].

\section{Health-Promoting Effects: Clinical Trial Findings}

5.1. Anti-Inflammatory Effect. Earlier literature reported that the administration of $1340 \mathrm{mg}$ of powdered extract of $U$. dioica (nettle leaves) reduced arthritis to half. A randomized control trial in 50 patients suffering from a chronic joint disease in Germany demonstrated the effectiveness of a combination of stewed nettle along with $50 \mathrm{mg}$ of diclofenac treatment (group $\mathrm{D} 50+\mathrm{U})$ compared to a standard dose of diclofenac $(200 \mathrm{mg})$ $[127,128]$. Results of this study indicated that both treatments were equally effective in mitigating clinical symptoms occurring due to acute arthritis. These results are of great importance for patients who suffer from nonsteroidal anti-inflammatory drugs (NSAIDs) intolerance because of ulceration or other gastric problems. However, further studies are required to find out whether nettle could be effective in the absence of NSAIDs $[127,129]$.

5.2. Diuretic Effect. In a study aiming to assess the impact of $15 \mathrm{~mL}$ nettle herb juice for treating myocardial or chronic insufficiency, 32 patients received 3 times daily such preparation in an open 2-week study. Later, the frequency of dosing was reduced to once a day in the morning. The daily volume of urine was increased significantly throughout the treatment. The patients with myocardial insufficiency in the $2^{\text {nd }}$ day of treatment was $9.2 \%$ higher $(p \leq 0.0005)$ than the baseline and patients of chronic venous insufficiency reported $23.9 \%$ higher $(p \leq 0.05)$ urine volume. Patients' weight (about 1\%) and systolic blood pressure showed slight decreases. Apart from slight side effects, like diarrhea, serum parameters remained stable and treatments were smoothly tolerated. Additionally, diuretic and natriuretic effects were detected, implying a renal function effect [127, 130]. Some objective indicators in this clinical investigation indicated statistically significant improvement, despite the small number of patients and the short duration of the study limiting the establishment of solid conclusions.

5.3. Antiallergic Effect. The safest remedy for allergy and sinus treatment is nettle. Indeed, it has been reported used in various ailments ranging from allergic rhinitis to hypertension. Lyophilized leaves of nettle have been clinically proven to relieve allergy symptoms [89, 113]. For example, a double-blind, randomized study was conducted with 98 individuals to try the effect of freeze-dried $U$. dioica herb (2 times $300 \mathrm{mg}$ ) on allergic rhinitis. After one week of therapy, daily symptom diaries and global response documented after follow-up were considered for assessment $[131,132]$. In the overall evaluations, $U$. dioica was ranked higher than placebo, and when the diary data were compared, U. dioica was just marginally higher [132]. Thus, even if the $U$. dioica trial appears to be effective, more research with a bigger and better-matched sample size and possibly a longer treatment period might be beneficial. Research into the mechanism of action of $U$. dioica and its potential for application in other allergy disorders is also recommended.

5.4. Antidiabetic Effect. The health benefit of the hydroalcoholic extract of $U$. dioica on blood lipids, hepatic enzymes, and nitric oxide levels was investigated in a randomized control trial, including 50 women with type 2 diabetes. $U$. dioica significantly decreased FPG and TG and increased SGPT levels and HDL, NO, and SOD levels compared to the control group after 8 weeks of treatment. This result supports using the hydroalcoholic extract of $U$. dioica as an antioxidant agent for additional therapy of diabetes to minimize complications, such as cardiovascular risk factors in diabetic patients [133]. However, the relatively small sample size and the lack of exact diet and exercise management of patients who participated in the study make the findings suggestive rather than conclusive. Therefore, trials with a larger number of patients and a longer intervention period are recommended to better understand $U$. dioica's benefits in diabetic patients.

Overall, these clinical studies are not appropriate for traditional use in indications, like the acute attack of chronic joint disease, myocardial or chronic venous insufficiency, and allergic rhinitis. Indeed, only the well-established use can be relevant in these indications; however, they are hardly good enough and the results of these trials cannot be used [134].

Since a small number of participants were included in the studies and were not double-blind (except Mittman's study) and that data are not detailed enough, the 
consequences are not influential. These studies may only support the authenticity of diuretic and anti-inflammatory effects; in this way, the traditional indications may be supported by them.

\section{Safety, Drug-Drug Interaction, and Adverse Effect of the Genus Urtica}

Though sweating and gastric discomfort are reported in some cases, the Urtica plant usually causes skin irritation upon touching it [135].

Hypersensitivity cases have been reported in patients with renal ailments $[33,130]$. When the hairs or spines on the stems and leaves of the stinging nettle come into contact with the skin, various physiologically active chemicals are released within seconds and in turn induce irritation, dermatitis, and urticaria [136]. These findings imply that histamine, which is released by the nettle, has a role in the rapid reaction to nettle stings. Moreover, the endurance of the stinging sensation, on the other hand, could indicate that there are chemicals in nettle fluid that are directly harmful to nerves or that can cause the subsequent release of other mediators [137]. Furthermore, urine flow is enhanced by the aerial parts of Urtica; hence, it is advised to inform the healthcare provider of whether the patient suffers from diabetes or kidney problems $[33,130]$. Urtica aerial parts at $1.25 \mathrm{~g} / \mathrm{kg}$ decline blood sugar following intake [135] and may potentiate concurrent antidiabetics' effect, high or low blood pressure [138-140].

Furthermore, the key underlying processes of this food plant and its phytonutrients in the management of urolithiasis include a diuretic effect, which can exacerbate the diuretic therapy in patients with renal disorders. Though nettle is reputed to be an abortifacient and to affect the menstrual cycle in traditional medicine, oral administration of $250 \mathrm{mg} / \mathrm{kg}$ of nettle to mice is devoid of antifertility activity. In the absence of clear evidence of antifertility potency, Urtica spp. should be completely avoided during pregnancy or in breastfeeding women and children [127]. For sure, Urtica dioica and Urtica urens preparations have been used orally as a postpartum "tonic" for treating anemia in nursing mothers and is a purported galactagogue. Still, no scientifically valid clinical trials support the safety and efficacy in nursing mothers or infants for any use [141].

Urtica dioica is used as an anti-inflammatory in rheumatoid arthritis. The anti-inflammatory effect of Urtica extract is due to its inhibitory effect on NF-kappaB activation and the genetic transcription factor that activates TNF- $\alpha$ and IL-1B in synovial tissue that lines the joint, lowering TNF- $\alpha$ and other inflammatory cytokines levels $[123,142]$. Therefore, Urtica spp. should be avoided in the case of acute arthritis due to the risk of drug-drug interaction [138].

Urtica spp. has also been reported to enhance the impact of CNS depressant medications [138]. The concomitant use of Urtica aerial parts with sedatives, including lorazepam (Ativan), phenobarbital (Donnatal), clonazepam (Klonopin), zolpidem (Ambien), and others may lead to sleepiness and drowsiness [127].

\section{Conclusions and Future Perspectives}

In short, while summarizing the ethnopharmacological reports on the use of Urtica species, $U$. dioica emerged as the most reported species, providing a rich source of active principles for developing novel treatment strategies. Despite its ancient use by people from different cultures and in different regions for the treatment of various ailments, the current achievements have stated that Urtica spp. have renowned pharmacological potentialities, including anti-inflammatory, anticancer, antioxidant, antidiabetic, antimicrobial, and antiviral effects that correlate, by one hand, with some traditional uses and, on the other hand, with the bioactive phytochemicals present, including phenolic compounds and terpenoids that may be effectively applied for preventive or therapeutic purposes in communicable and noncommunicable diseases. However, there is still a large gap in in vivo experiments and clinical trials using plant-based preparations or isolated phytochemicals from Urtica spp. that need to be filled in a short time so that new windows for preventive, therapeutic, and agroindustrial purposes can be open.

\section{Data Availability}

The data supporting this review were taken from previously reported studies and datasets, which have been cited. The processed data are available from the corresponding author upon request.

\section{Conflicts of Interest}

The authors have no relevant affiliations or financial involvement with any organization or entity with a financial interest in or financial conflict with the subject matter or materials discussed in the manuscript. This includes employment, consultancies, honoraria, stock ownership or options, expert testimony, grants or patents received or pending, or royalties.

\section{Authors' Contributions}

All the authors made a significant contribution to the work reported, whether that is in the conception, study design, execution, acquisition of data, analysis, and interpretation, or in all these areas: that is, revising or critically reviewing the article; giving final approval of the version to be published; agreeing on the journal to which the article has been submitted; and confirming to be accountable for all aspects of the work. All the authors have read and agreed to the published version of the manuscript.

\section{References}

[1] K. M. Ahmed and S. J. S. R. I. P. Parsuraman, "Urtica dioica L.,(Urticaceae): a stinging nettle," Systematic Reviews in Pharmacy, vol. 5, no. 1, p. 6, 2014.

[2] D. Kregiel, E. Pawlikowska, and H. Antolak, "Urtica spp: ordinary plants with extraordinary properties," Molecules, vol. 23, no. 7, p. 1664, 2018. 
[3] J. Mutke, R. Jacobs, K. Meyers, T. Henning, and M. Weigend, "Diversity patterns of selected andean plant groups correspond to topography and habitat dynamics, not orogeny," Frontiers in Genetics, vol. 5, no. 351, p. 351, 2014.

[4] M. Weigend and A. K. Monro, "Urtica L. (Urticaceae)," in Flora Mesoamericana, pp. 171-174, Missouri Botanical Garden Press, St. Louis, MO, USA, 2015.

[5] G. Hashimoto, "Brazil," in Biology and Ecology of Weeds, M. Numata and W. Holzner, Eds., The Hague: W. Junk: Kluwer Academic, Hague, Netherlands, pp. 333-337, 1982.

[6] M. Srlltek and M. Teckelmann, "Review of biology and ecology of Urtica dioica," Preslia Praha, vol. 70, pp. 1-19, 1998.

[7] B. Grosse-Veldmann and M. Weigend, "Weeding the nettles III: named nonsense versus named morphotypes in European Urtica dioica L. (Urticaceae)," Phytotaxa, vol. 208, no. 4, p. 239, 2015.

[8] B. Grosse-Veldmann, B. J. Conn, and M. Weigend, "Weeding the nettles IV: a redefinition of Urtica incisa and allies in New Zealand and Australia, including the segregation of two new species Urtica sykesii and U. perconfusa," Phytotaxa, vol. 245, no. 4, p. 251, 2016.

[9] B. Grosse-Veldmann, N. M. Nürk, R. Smissen, I. Breitwieser, D. Quandt, and M. Weigend, "Pulling the sting out of nettle systematics-a comprehensive phylogeny of the genus Urtica L. (Urticaceae)," Molecular Phylogenetics and Evolution, vol. 102, pp. 9-19, 2016.

[10] M. Weigend, A. Cano, and E. F. Rodríguez, "New species and new records of the flora in amotape-huancabamba zone: endemics and biogeographic limits," Revista Peruana de Biologia, vol. 12, no. 2, pp. 249-274, 2005.

[11] A. Kumar, A. Singh Bisht, S. Joshi, and D. Juyal, "Pharmacognostical and phytochemical study of a plant Urtica parviflora Roxb.-a review," Journal of Pharmacognosy and Phytochemistry, vol. 6, no. 3, pp. 42-45, 2017.

[12] Y. Yeşil and İ. İnal, "Traditional knowledge of wild edible plants in hasankeyf (Batman Province, Turkey)," Acta Societatis Botanicorum Poloniae, vol. 88, no. 3, 2019.

[13] Y. Yesil, M. Celik, and B. Yilmaz, "Wild edible plants in yesilli (Mardin-Turkey), a multicultural area," Journal of Ethnobiology and Ethnomedicine, vol. 15, no. 1, p. 52, 2019.

[14] F. Varga, I. Šolić, M. J. Dujaković, Ł. Łuczaj, and M. Grdiša, "The first contribution to the ethnobotany of inland dalmatia: medicinal and wild food plants of the Knin area, Croatia," Acta Societatis Botanicorum Poloniae, vol. 88, no. 2, 2019.

[15] A. A. Samaha, M. Fawaz, A. Salami, S. Baydoun, and A. H. Eid, "Antihypertensive indigenous lebanese plants: ethnopharmacology and a clinical trial," Biomolecules, vol. 9, no. 7, p. 292, 2019.

[16] Ş. Kültür, G. Melikoglu, S. Kurtoglu, and O. Altınbaşak, "Türkiye de mide ülserinde kullanılan tıbbi bitkiler," Marmara Pharmaceutical Journal, vol. 22, no. 1, pp. 1-14, 2018.

[17] I. Vitasovic Kosic, J. Juracak, and L. Luczaj, "Using ellenbergpignatti values to estimate habitat preferences of wild food and medicinal plants: an example from northeastern Istria (Croatia)," Journal of Ethnobiology and Ethnomedicine, vol. 13, no. 1, p. 31, 2017.

[18] Ç. Kizilarslan Hançer, E. Sevgi, B. Büyükkılıç, E. Çakir, and M. Akkaya, "Traditional knowledge of wild edible plants of biga (çanakkale), Turkey," Acta Societatis Botanicorum Poloniae, vol. 89, no. 1, 2020.

[19] M. Ibrahim, K. Rehman, A. Razzaq et al., "Investigations of phytochemical constituents and their pharmacological properties isolated from the genus Urtica: critical review and analysis," Critical Reviews in Eukaryotic Gene Expression, vol. 28, no. 1, pp. 25-66, 2018.

[20] İ. Gürbüz, A. Gençler Ozkan, T. Günbatan, F. Demirci, and E. Yesilada, "Folk medicine in düzce province (Turkey)," Turkish Journal of Botany, vol. 43, no. 6, pp. 769-784, 2019.

[21] P. M. Guarrera and V. Savo, "Wild food plants used in traditional vegetable mixtures in Italy," Journal of Ethnopharmacology, vol. 185, pp. 202-234, 2016.

[22] U. Koca, C. Aka Dönmez, and M. G. Oz, "Plants used in anatolian traditional medicine for the treatment of hemorrhoid," Records of Natural Products, vol. 11, pp. 235-250, 2017.

[23] K. Dolina, M. Jug-Dujaković, Ł. Łuczaj, and I. VitasovićKosić, "A century of changes in wild food plant use in coastal croatia: the example of krk and poljica," Acta Societatis Botanicorum Poloniae, vol. 85, no. 3, pp. 3508-3529, 2016.

[24] H. Bhatia, Y. P. Sharma, R. K. Manhas, and K. Kumar, "Traditionally used wild edible plants of district Udhampur, J\&K, India," Journal of Ethnobiology and Ethnomedicine, vol. 14, p. 73, 2018.

[25] M. Asadi-Samani, M.-T. Moradi, L. Mahmoodnia, S. Alaei, F. Asadi-Samani, and T. Luther, "Traditional uses of medicinal plants to prevent and treat diabetes; an updated review of ethnobotanical studies in Iran," Journal of Nephropathology, vol. 6, no. 3, pp. 118-125, 2017.

[26] M. S. Abu-Darwish and T. Efferth, "Medicinal plants from near east for cancer therapy," Frontiers in Pharmacology, vol. 9, no. 56, p. 56, 2018.

[27] J. Sharifi-Rad, C. Quispe, J. Herrera-Bravo et al., "Phytochemical constituents, biological activities, and health-promoting effects of the melissa officinalis," Oxidative Medicine and Cellular Longevity, vol. 2021, Article ID 6584693, 20 pages, 2021.

[28] R. Hossain, C. Quispe, J. Herrera-Bravo et al., "Lasia spinosa chemical composition and therapeutic potential: a literaturebased review," Oxidative Medicine and Cellular Longevity, vol. 2021, Article ID 1602437, 12 pages, 2021.

[29] M. Hejcman, M. Češková, J. Schellberg, and S. Pätzold, “The rengen grassland experiment: effect of soil chemical properties on biomass production, plant species composition and species richness," Folia Geobotanica, vol. 45, no. 2, pp. 125-142, 2010.

[30] C. D. Pigott and K. Taylor, "The distribution of some woodland herbs in relation to the supply of nitrogen and phosphorus in the soil," The Journal of Animal Ecology, vol. 33, p. 175, 1964.

[31] C. Olsen, "The ecology of urtica dioica," The Journal of Ecology, vol. 9, no. 1, pp. 1-18, 1921.

[32] G. Corsi and A. Masini, Anatomical and Ecological Aspects in Italian Taxa of the Genus Urtica, Dipartimento di Scienze Botaniche, University of Pisa, Pisa, Italy, 1997.

[33] L. Testai, S. Chericoni, V. Calderone et al., "Cardiovascular effects of Urtica dioica L. (Urticaceae) roots extracts: in vitro and in vivo pharmacological studies," Journal of Ethnopharmacology, vol. 81, no. 1, pp. 105-109, 2002.

[34] I. Friis, "Urticaceae," in Flowering Plants Dicotyledons, K. Kubitzki, J. G. Rohwer, and V. Bittrich, Eds., Springer, Berlin, Germany, pp. 612-630, 1993.

[35] D. Geltman, "Urtica galeopsifolia wierzb. ex opiz (Urticaceae) in wicken Fen (E. England)," Watsonia, vol. 19, 1992.

[36] E. Hultén and M. Fries, Atlas of North European Vascular Plants, North of the Tropic of Cancer, North Lungsai, Mizoram, 1986. 
[37] J. P. Grime, J. G. Hodgson, and R. Hunt, Comparative Plant Ecology: A Functional Approach to Common British Species, Springer, Berlin, Germany, 1989.

[38] C. Stefanescu, "The nature of migration in the red admiral butterfly vanessa atalanta: evidence from the population ecology in its southern range," Ecological Entomology, vol. 26, no. 5, pp. 525-536, 2001.

[39] P. B. Hardy and R. L. H. Dennis, "The impact of urban development on butterflies within a city region," Biodiversity and Conservation, vol. 8, no. 9, pp. 1261-1279, 1999.

[40] S. Bryant, C. Thomas, and J. Bale, "Nettle-feeding nymphalid butterflies: temperature, development and distribution," Ecological Entomology, vol. 22, no. 4, pp. 390-398, 1997.

[41] B. N. K. Davis, "The European distribution of insects on stinging nettles, Urtica dioica L: a field survey," Bolletino di Zoologia, vol. 56, no. 4, pp. 321-326, 1989.

[42] S. R. Bryant, C. D. Thomas, and J. S. Bale, "Thermal ecology of gregarious and solitary nettle-feeding nymphalid butterfly larvae," Oecologia, vol. 122, no. 1, pp. 1-10, 2000.

[43] J. Sharifi-Rad, A. Bahukhandi, P. Dhyani et al., "Therapeutic potential of neoechinulins and their derivatives: an overview of the molecular mechanisms behind pharmacological activities," Frontiers in Nutrition, vol. 8, Article ID 664197, 2021.

[44] J. Sharifi-Rad, C. Quispe, J. Herrera-Bravo et al., "A pharmacological perspective on plant-derived bioactive molecules for epilepsy," Neurochemical Research, vol. 46, no. 9, 2021.

[45] F. Abe, "The CDF detector: an overview," Nuclear Instruments and Methods in Physics Research Section A: Accelerators, Spectrometers, Detectors and Associated Equipment, vol. 271, no. 3, pp. 387-403, 1988.

[46] H. Aishan, M. Baba, N. Iwasaki, H. Kuang, and T. Okuyama, "The constituents of Urtica cannabinaused in uighur medicine," Pharmaceutical Biology, vol. 48, no. 5, pp. 577-583, 2010.

[47] P. Akbay, A. A. Basaran, U. Undeger, and N. Basaran, "In vitro immunomodulatory activity of flavonoid glycosides from Urtica dioica L," Phytotherapy Research, vol. 17, no. 1, pp. 34-37, 2003.

[48] J. Asgarpanah and R. Khoshkam, "Phytochemistry and pharmacologic properties of Urtica dioica L," Journal of Medicinal Plants Research, vol. 6, 2012.

[49] H. Bhatia, R. K. Manhas, K. Kumar, and R. Magotra, "Traditional knowledge on poisonous plants of Udhampur district of Jammu and Kashmir, India," Journal of Ethnopharmacology, vol. 152, no. 1, pp. 207-216, 2014.

[50] A. N. Binns, R. H. Chen, H. N. Wood, and D. G. Lynn, "Cell division promoting activity of naturally occurring dehydrodiconiferyl glucosides: do cell wall components control cell division?" Proceedings of the National Academy of Sciences, vol. 84, no. 4, pp. 980-984, 1987.

[51] M. Ekor, "The growing use of herbal medicines: issues relating to adverse reactions and challenges in monitoring safety," Frontiers in Pharmacology, vol. 4, p. 177, 2014.

[52] M. A. Farag, M. Weigend, F. Luebert, G. Brokamp, and L. A. Wessjohann, "Phytochemical, phylogenetic, and antiinflammatory evaluation of 43 Urtica accessions (stinging nettle) based on UPLC-Q-TOF-MS metabolomic profiles," Phytochemistry, vol. 96, pp. 170-183, 2013.

[53] B. Feng, X. Yan, H. Wang, L. Shi, L. Tang, and Y. Wang, "Two new secolignan glycosides from the roots of Urtica triangularis hand.-mazz," Fitoterapia, vol. 81, no. 6, pp. 607-609, 2010.
[54] S. Kumar, S. Ahmad, and S. Harikumar, "Pharmacognostical and pharmacological studies on urtica parviflora roxb. A review," International Journal of Universal Pharmacy and Bio Sciences, vol. 3, no. 4, pp. 179-184, 2014.

[55] E. S. Lapinskaya, Y. F. Kopyt'ko, E. A. Timokhina et al., "Amino acids and cyclic dipeptides in stinging nettle (Urtica dioica and U. urens) homeopathic matrix tinctures," Pharmaceutical Chemistry Journal, vol. 42, no. 11, pp. 650-653, 2009.

[56] J. Ma, X. Jin, L. Yang, and Z. L. Liu, "Diarylheptanoids from the rhizomes of zingiber officinale," Phytochemistry, vol. 65, no. 8, pp. 1137-1143, 2004.

[57] C. Rice-Evans, N. Miller, and G. Paganga, "Antioxidant properties of phenolic compounds," Trends in Plant Science, vol. 2, no. 4, pp. 152-159, 1997.

[58] M. Tekin, H. Özbek, and A. Him, "Investigation of acute toxicity, anti-inflammatory and analgesic effect of Urtica dioica L," Pharmacologyonline, vol. 1, pp. 1210-1215, 2009.

[59] N. A. Yunuskhodzhaeva, V. N. Abdullabekova, K. S. Ibragimova, and L. G. Mezhlumyan, "Amino-acid composition of Urtica dioica leaves and Polygonum hydropiper and P. aviculare herbs," Chemistry of Natural Compounds, vol. 50, no. 5, pp. 970-971, 2014.

[60] R. Dhouibi, H. Affes, M. Ben Salem et al., "Ksouda, Screening of pharmacological uses of Urtica dioica and others benefits," Progress in Biophysics and Molecular Biology, pp. 67-77, 2020.

[61] S. Sitrallah and J. Merza, "Chemical composition of essential oil extracted from urtica pilulifera and evaluation its biological activity," Food Science and Quality Management, vol. 78, pp. 44-48, 2018.

[62] D. C. Ilies, I. Tudor, and V. Radulescu, "Chemical composition of the essential oil of Urtica dioica," Chemistry of Natural Compounds, vol. 48, no. 3, pp. 506-507, 2012.

[63] X. Luo, L.-L. Li, S.-S. Zhang et al., "Therapeutic effects of total coumarins from Urtica dentata Hand on collagen-induced arthritis in Balb/c mice," Journal of Ethnopharmacology, vol. 138, no. 2, pp. 523-529, 2011.

[64] T. Ozen and H. Korkmaz, "Modulatory effect of Urtica dioica L. (Urticaceae) leaf extract on biotransformation enzyme systems, antioxidant enzymes, lactate dehydrogenase and lipid peroxidation in mice," Phytomedicine, vol. 10, no. 5, pp. 405-415, 2003.

[65] D. M. Abo-elmatty, S. S. Essawy, J. M. Badr, and O. Sterner, "Antioxidant and anti-inflammatory effects of Urtica pilulifera extracts in type2 diabetic rats," Journal of Ethnopharmacology, vol. 145, no. 1, pp. 269-277, 2013.

[66] A. Bayrami, S. Haghgooie, S. R. Pouran, F. M. Arvanag, and A. Habibi-Yangjeh, "Synergistic antidiabetic activity of $\mathrm{ZnO}$ nanoparticles encompassed by Urtica dioica extract," Advanced Powder Technology, vol. 31, no. 5, 2020.

[67] M. Ilhan, Z. Ali, I. A. Khan, H. Taştan, and E. Küpeli Akkol, "Bioactivity-guided isolation of flavonoids from Urtica dioica L. and their effect on endometriosis rat model," Journal of Ethnopharmacology, vol. 243, Article ID 112100, 2019.

[68] Q. Zhang, L. Li, L. Liu et al., "Effects of the polysaccharide fraction of Urtica fissa on castrated rat prostate hyperplasia induced by testosterone propionate," Phytomedicine, vol. 15, no. 9 , pp. $722-727,2008$.

[69] S. A. Nadia, "Effect of nettle (Urtica dioica) extract on gentamicin induced nephrotoxicity in male rabbits," Asian Pacific Journal of Tropical Biomedicine, vol. 9, pp. 729-732, 2015. 
[70] Y. Kumaki, M. K. Wandersee, A. J. Smith et al., "Inhibition of severe acute respiratory syndrome coronavirus replication in a lethal SARS-CoV BALB/c mouse model by stinging nettle lectin, Urtica dioica agglutinin," Antiviral Research, vol. 90, no. 1, pp. 22-32, 2011.

[71] E. Hwang, H. T. T. Ngo, S. A. Seo et al., "Urtica thunbergiana prevents UVB-induced premature skin aging by regulating the transcription factor NFATc1: an in vitro and in vivo study," Journal of Functional Foods, vol. 36, pp. 162-177, 2017.

[72] A. Mohammadi, B. Mansoori, P. C. Baradaran et al., "Urtica dioica extract inhibits proliferation and induces apoptosis and related gene expression of breast cancer cells in vitro and in vivo," Clinical Breast Cancer, vol. 17, no. 6, pp. 463-470, 2017.

[73] R. E. Uncini Manganelli, L. Zaccaro, and P. E. Tomei, "Antiviral activity in vitro of Urtica dioica L., Parietaria diffusa M. et K. and Sambucus nigra L," Journal of Ethnopharmacology, vol. 98, no. 3, pp. 323-327, 2005.

[74] J. Balzarini, J. Neyts, D. Schols et al., "The mannose-specific plant lectins from cymbidium hybrid and epipactis helleborine and the ( $\mathrm{N}$-acetylglucosamine)n-specific plant lectin from Urtica dioica are potent and selective inhibitors of human immunodeficiency virus and cytomegalovirus replication in vitro," Antiviral Research, vol. 18, no. 2, pp. 191-207, 1992.

[75] Y. Taheri, N. Joković, J. Vitorović, O. Grundmann, A. Maroyi, and D. Calina, "The burden of the serious and difficult-to-treat infections and a new antibiotic available: cefiderocol," Frontiers in Pharmacology, vol. 11, p. 18, 2021.

[76] O. Zlatian, A. T. Balasoiu, M. Balasoiu et al., "Antimicrobial resistance in bacterial pathogens among hospitalised patients with severe invasive infections," Experimental and Therapeutic Medicine, vol. 16, no. 6, pp. 4499-4510, 2018.

[77] R. Singh, S. Hussain, R. Verma, and P. Sharma, "Anti-mycobacterial screening of five Indian medicinal plants and partial purification of active extracts of cassia sophera and Urtica dioica," Asian Pacific Journal of Tropical Medicine, vol. 6, no. 5, pp. 366-371, 2013.

[78] A. Modarresi-Chahardehi, D. Ibrahim, S. Fariza-Sulaiman, and L. Mousavi, "Screening antimicrobial activity of various extracts of Urtica dioica," Revista de Biologia Tropical, vol. 60, pp. 1567-1576, 2012.

[79] S. A. Dar, F. A. Ganai, A. R. Yousuf, M.-u.-H. Balkhi, T. M. Bhat, and P. Sharma, "Pharmacological and toxicological evaluation of Urtica dioica," Pharmaceutical Biology, vol. 51, no. 2, pp. 170-180, 2013.

[80] Z. Zeković, A. Cvetanović, J. Švarc-Gajić et al., "Chemical and biological screening of stinging nettle leaves extracts obtained by modern extraction techniques," Industrial Crops and Products, vol. 108, pp. 423-430, 2017.

[81] S. Zenão, A. Aires, C. Dias, M. Saavedra, and C. Fernandes, "Antibacterial potential of Urtica dioica and Lavandula angustifolia extracts against methicillin resistant staphylococcus aureus isolated from diabetic foot ulcers," Journal of Herbal Medicine, vol. 10, pp. 53-58, 2017.

[82] L. Ommar, "The anthelmintic effect of urtica dioica and tanacetum vulgare L. On protoscoleces of echinococcus granulosus," International Journal of Sciences: Basic and Applied Research (IJSBAR), vol. 11, no. 1, pp. 84-89, 2013.

[83] M. Sarma Kataki, V. Murugamani, A. Rajkumari, P. Mehra, D. Awasthi, and R. Yadav, "Antioxidant, hepatoprotective, and anthelmintic activities of methanol extract of urtica dioica L. Leaves," Pharmaceutical Crops, vol. 3, no. 1, 2012.
[84] J. Sharifi-Rad, C. Quispe, J. Kumar Patra et al., "Paclitaxel: application in modern oncology and nanomedicine-based cancer therapy," Oxidative Medicine and Cellular Longevity, vol. 2021, Article ID 3687700, 24 pages, 2021.

[85] J. Sharifi-Rad, C. Quispe, M. Butnariu et al., "Chitosan nanoparticles as a promising tool in nanomedicine with particular emphasis on oncological treatment," Cancer Cell International, vol. 21, no. 1, p. 318, 2021.

[86] B. Mansoori, A. Mohammadi, S. Hashemzadeh et al., "Urtica dioica extract suppresses miR-21 and metastasis-related genes in breast cancer," Biomedicine \& Pharmacotherapy, vol. 93, pp. 95-102, 2017.

[87] S. Rizk, N. Al Bast, M. H. Hodroj, and J. Borjac, “Aqueous urtica dioica leaves extract inhibits proliferation of acute myeloid leukemia cells in vitro," Clinical Lymphoma Myeloma and Leukemia, vol. 17, pp. S279-S280, 2017.

[88] S. Fattahi, A. M. Ardekani, E. Zabihi et al., "Antioxidant and apoptotic effects of an aqueous extract of Urtica dioica on the MCF-7 human breast cancer cell line," Asian Pacific Journal of Cancer Prevention, vol. 14, no. 9, pp. 5317-5323, 2013.

[89] A. R. Carvalho, G. Costa, A. Figueirinha et al., "Urtica spp: phenolic composition, safety, antioxidant and anti-inflammatory activities," Food Research International, vol. 99, no. 1, pp. 485-494, 2017.

[90] A. O. Docea, D Calina, A. M Buga et al., "The effect of silver nanoparticles on antioxidant/pro-oxidant balance in a murine model," International Journal of Molecular Sciences, vol. 21, no. 4, p. 17, 2020.

[91] E. A. Guzmán Hernández, "Renoprotective and hepatoprotective effects of hippocratea excelsa on metabolic syndrome in fructose-fed rats," Farmacia, vol. 68, no. 6, pp. 1106-1119, 2020.

[92] C. Bourgeois, É. A. Leclerc, C. Corbin et al., "Nettle (Urtica dioica L.) as a source of antioxidant and anti-aging phytochemicals for cosmetic applications," Comptes Rendus Chimie, vol. 19, no. 9, pp. 1090-1100, 2016.

[93] A. Basso, C. Marrassini, C. Anesini, and S. Gorzalczany, "Acute hypotensive, diuretic and antioxidant activities induced by urtica circularis," European Journal of Medicinal Plants, vol. 14, no. 2, pp. 1-9, 2016.

[94] K. Ghaima, N. M. Hashim, and S. A. Ali, "Antibacterial and antioxidant activities of ethyl acetate extract of nettle (Urtica dioica) and dandelion (Taraxacum officinale)," Journal of Applied Pharmaceutical Science, vol. 3, pp. 96-99, 2013.

[95] M. Mzid, S. Ben Khedir, S. Bardaa, Z. Sahnoun, and T. Rebai, "Chemical composition, phytochemical constituents, antioxidant and anti-inflammatory activities of Urtica urens L. leaves," Archives of Physiology and Biochemistry, vol. 123, no. 2, pp. 93-104, 2017.

[96] S. Pandey, S. P. Sah, M. L. Sah, and D. Mishra, "An antioxidant potential of hydromethanolic extract of urtica parviflora roxb," Journal of Basic and Clinical Pharmacy, vol. 1, no. 3, pp. 191-195, 2010.

[97] C. Scheau, C. Caruntu, I. A. Badarau et al., "Cannabinoids and inflammations of the gut-lung-skin barrier," Journal of Personalized Medicine, vol. 11, no. 6, 2021.

[98] M. El Haouari, M. Bnouham, M. Bendahou et al., "Inhibition of rat platelet aggregation by Urtica dioica leaves extracts," Phytotherapy Research, vol. 20, no. 7, pp. 568-572, 2006.

[99] M. Sifaki, D. Calina, A. O. Docea et al., "A novel approach regarding the anti-aging of facial skin through collagen reorganization," Experimental and Therapeutic Medicine, vol. 19, no. 1, pp. 717-721, 2020. 
[100] I. Turel, O. Yilmaz, E. Ayaz, G. Oto, and U. Mercan, “Anthelmintic activity of Urtica dioica L. in mice naturally infected with aspiculuris tetraptera," Journal of Animal and Veterinary Advances, vol. 1, no. 12, pp. 1628-1630, 2008.

[101] A. M. Iordache, A. M. Buga, D Albulescu et al., "Phosphodiesterase-5 inhibitors ameliorate structural kidney damage in a rat model of contrast-induced nephropathy," Food and Chemical Toxicology, vol. 143, Article ID 111535, 2020.

[102] A. M. Kamal, "Double therapy with pegylated interferon and ribavirin for chronic hepatitis c. a pharmacogenetic guide for predicting adverse eventS," Farmacia, vol. 65, no. 6, pp. 877-884, 2017.

[103] M. T. Islam, C. Quispe, D. M. El-Kersh et al., "A literaturebased update on benincasa hispida (thunb.) cogn: traditional uses, nutraceutical, and phytopharmacological profiles," Oxidative Medicine and Cellular Longevity, vol. 2021, Article ID 6349041, 19 pages, 2021.

[104] G. Avci, E. Kupeli, A. Eryavuz, E. Yesilada, and I. Kucukkurt, "Antihypercholesterolaemic and antioxidant activity assessment of some plants used as remedy in Turkish folk medicine," Journal of Ethnopharmacology, vol. 107, no. 3, pp. 418-423, 2006.

[105] M. Nassiri-Asl, F. Zamansoltani, E. Abbasi, M.-M. Daneshi, and A.-A. Zangivand, "Effects of Urtica dioica extract on lipid profile in hypercholesterolemic rats," Journal of Chinese Integrative Medicine, vol. 7, no. 5, pp. 428-433, 2009.

[106] N. R. Barman, S. Nandy, R. Datta, and P. K. Kar, "Cardioprotective effect of ethanolic extract of Urtica parviflora roxb. against isoproterenol induced myocardial infarction in rats," Indian Journal of Pharmacology, vol. 45, no. 5, pp. 513-516, 2013.

[107] A. Legssyer, A. Ziyyat, H. Mekhfi et al., "Cardiovascular effects of Urtica dioica L. in isolated rat heart and aorta," Phytotherapy Research, vol. 16, no. 6, pp. 503-507, 2002.

[108] D. Shackebaei, A. Godini, M. Abolghazi, M. B. Majnouni, and M. Hesari, "Protection of ischemic and reperfused rat heart by aqueous extract of urtica dioica," Iranian Cardiovascular Research Journal, vol. 4, pp. 107-111, 2010.

[109] G. A. Soliman, "The potential cardioprotective effect of matricaria chamomilla extract against diabetes-induced oxidative stress in rats," Farmacia, vol. 68, no. 2, pp. 269-279, 2020.

[110] A. Ahangarpour, M. Mohammadian, and M. Dianat, "Antidiabetic effect of hydroalcholic urticadioica leaf extract in male rats with fructose-induced insulin resistance," Iranian Journal of Medical Sciences, vol. 37, no. 3, pp. 181-186, 2012.

[111] G. Kavalali, H Tuncel, S Göksel, and H. H Hatemi, "Hypoglycemic activity of Urtica pilulifera in streptozotocindiabetic rats," Journal of Ethnopharmacology, vol. 84, no. 2-3, pp. 241-245, 2003.

[112] H. Zhang, "Analysis of the polysaccharides from Urtica angustifolia and their anti-fatigue activity," African Journal of Pharmacy and Pharmacology, vol. 7, no. 22, pp. 1438-1447, 2013.

[113] I. Gülçin, O. I. Küfrevioglu, M. Oktay, and M. E. Büyükokuroglu, "Antioxidant, antimicrobial, antiulcer and analgesic activities of nettle (Urtica dioica L.)," Journal of Ethnopharmacology, vol. 90, no. 2-3, pp. 205-215, 2004.

[114] N. Jaradat, "Standardization the crude extracts of all urtica plant species growing in Palestine for quality control of cosmeceutical and pharmaceutical Formulations," International Journal of Pharmaceutical and Clinical Research, vol. 7, pp. 368-373, 2015.
[115] W. Wang, X. G. Yan, L. X. Duan et al., "Two new secolignans from the roots of Urtica mairei Levl," Chinese Chemical Letters, vol. 19, no. 10, pp. 1212-1214, 2008.

[116] R. W. Hartmann, M. Mark, and F. Soldati, "Inhibition of 5 $\alpha$-reductase and aromatase by PHL-00801 (Prostatonin), a combination of PY102 (Pygeum africanum) and UR102 (Urtica dioica) extracts," Phytomedicine, vol. 3, no. 2, pp. 121-128, 1996.

[117] V. Khare, P. Kushwaha, S. Verma, A. Gupta, S. Srivastava, and A. Rawat, "Pharmacognostic evaluation and antioxidant activity of urtica dioica L," Chinese Medicine, vol. 03, no. 03, pp. 128-135, 2012.

[118] S. Gorzalczany, C. Marrassini, J. Miño, C. Acevedo, and G. Ferraro, "Antinociceptive activity of ethanolic extract and isolated compounds of Urtica circularis," Journal of Ethnopharmacology, vol. 134, no. 3, pp. 733-738, 2011.

[119] C. Marrassini, C. Acevedo, J. Miño, G. Ferraro, and S. Gorzalczany, "Evaluation of antinociceptive, antinflammatory activities and phytochemical analysis of aerial parts of Urtica urens L," Phytotherapy Research, vol. 24, no. 12, pp. 1807-1812, 2010.

[120] Z. Doukkali, K. Taghzouti, E. H. Bouidida, M. Nadjmouddine, Y. Cherrah, and K. Alaoui, "Evaluation of anxiolytic activity of methanolic extract of Urtica urens in a mice model," Behavioral and Brain Functions, vol. 11, no. 1, p. 19, 2015.

[121] Z. Yongna, R. Wantana, B. Pisit, L. Zhongkun, and Z. Rongping, "Analgesic and antipyretic activities of the aqueous extract of Urtica macrorrhiza in experimental animals," Fitoterapia, vol. 76, no. 1, pp. 91-95, 2005.

[122] C. Marrassini, R. Davicino, C. Acevedo, C. Anesini, S. Gorzalczany, and G. Ferraro, "Vicenin-2, a potential antiinflammatory constituent of Urtica circularis Journal of Natural Products, vol. 74, no. 6, pp. 1503-1507, 2011.

[123] K. Riehemann, B. Behnke, and K. Schulze-Osthoff, "Plant extracts from stinging nettle (Urtica dioica), an antirheumatic remedy, inhibit the proinflammatory transcription factor NF- $\kappa$ B,” FEBS Letters, vol. 442, no. 1, pp. 89-94, 1999.

[124] M. Xiang, S. Zhang, J. Lu et al., "Antilithic effects of extracts from Urtica dentata hand on calcium oxalate urinary stones in rats," Journal of Huazhong University of Science and Technology [Medical Sciences], vol. 31, no. 5, pp. 673-677, 2011.

[125] M Bnouham, "Antihyperglycemic activity of the aqueous extract of Urtica dioica," Fitoterapia, vol. 74, no. 7-8, pp. 677-681, 2003.

[126] B. Tan and J. Vanitha, "Immunomodulatory and antimicrobial effects of some traditional Chinese medicinal herbs: a review," Current Medicinal Chemistry, vol. 11, no. 11, pp. 1423-1430, 2004

[127] Z. Biró-Sándor, "Assessment report on Urtica dioica L., Urtica urens L., their hybrids or their mixtures, radix," Report, Committee on Herbal Medicinal Products (HMPC) Assessment, European Medicines Agency, Amsterdam, Netherlands, 2012.

[128] Chinese Rheumatism Data Center and Chinese Systemic Lupus Erythematosus Treatment and Research Group, "Recommendation for the prevention and treatment of nonsteroidal anti-inflammatory drug-induced gastrointestinal ulcers and its complications," Zhonghua Nei Ke Za Zhi, vol. 56, no. 1, pp. 81-85, 2017.

[129] S. Chrubasik, A. Schmidt, H. Junck, and M. Pfisterer, "Wirksamkeit und wirtschaftlichkeit von teufelskrallenwurzelextrakt bei rückenschmerzen: erste ergebnisse 
einer therapeutischen kohortenstudie," Complementary Medicine Research, vol. 4, no. 6, pp. 332-336, 1997.

[130] A. Tahri, S. Yamani, A. Legssyer et al., "Acute diuretic, natriuretic and hypotensive effects of a continuous perfusion of aqueous extract of Urtica dioica in the rat," Journal of Ethnopharmacology, vol. 73, no. 1-2, pp. 95-100, 2000.

[131] A. Kalia, B. Joshi, and M. Mukhija, "Pharmacognostical review of Urtica dioica L," International Journal of Green Pharmacy, vol. 8, no. 4, pp. 201-209, 2014.

[132] P. Mittman, "Randomized, double-blind study of freezedried Urtica dioica in the treatment of allergic rhinitis," Planta Medica, vol. 56, no. 1, pp. 44-47, 1990.

[133] A. Amiri Behzadi, H. Kalalian-Moghaddam, and A. H. Ahmadi, "Effects of Urtica dioica supplementation on blood lipids, hepatic enzymes and nitric oxide levels in type 2 diabetic patients: a double blind, randomized clinical trial," Avicenna Journal of Phytomedicine, vol. 6, no. 6, pp. 686-695, 2016.

[134] B. Sandor, "Assessment report on urttica dioica L., and urtica wens L., herba," Report, pp. 1-26, European Medicines Agency, Amsterdam, Netherlands, 2008.

[135] B. Farzami, D. Ahmadvand, S. Vardasbi, F. J. Majin, and S. Khaghani, "Induction of insulin secretion by a component of Urtica dioica leave extract in perifused islets of langerhans and its in vivo effects in normal and streptozotocin diabetic rats," Journal of Ethnopharmacology, vol. 89, no. 1, pp. 47-53, 2003.

[136] B. E. Anderson, C. J. Miller, and D. R. Adams, "Stinging nettle dermatitis," American Journal of Contact Dermatitis: Official Journal of the American Contact Dermatitis Society, vol. 14, no. 1, pp. 44-46, 2003.

[137] F. Oliver, E. U. Amon, A. Breathnach et al., "Contact urticaria due to the common stinging nettle (Urtica dioica)histological, ultrastructural and pharmacological studies," Clinical and Experimental Dermatology, vol. 16, no. 1, pp. 1-7, 1991.

[138] K. Kőszegi, J. Kocsis, G. Vatai, and E. Bekassy-Molnar, "Antimicrobial effects of the stinging nettle (urtica dioica L.)," Analecta Technica Szegedinensia, vol. 11, no. 2, pp. 10-15, 2017.

[139] N. Deye, F. Vincent, P. Michel et al., "Changes in cardiac arrest patients' temperature management after the 2013 "TTM" trial: results from an international survey," Annals of Intensive Care, vol. 6, no. 1, p. 4, 2016.

[140] G. M. Kavalali, Urtica: Therapeutic and Nutritional Aspects of Stinging Nettles, CRC, Boca Raton, FL, USA, 2003.

[141] Drugs and Lactation Database (LactMed), Stinging Nettle, National Library of Medicine, Bethesda, MD, USA, 2021.

[142] T. Teucher, B. Obertreis, T. Ruttkowski, and H. Schmitz, "Cytokine secretion in whole blood of healthy subjects following oral administration of Urtica dioica L. plant extract," Arzneimittelforschung, vol. 46, no. 9, pp. 906-910, 1996. 